\title{
The Long-term Illinois River Fish Population Monitoring Program
}

\author{
F-101-R \\ Final Report

\section{Todd M. Koel and Richard E. Sparks}

Illinois Natural History Survey

LTRMP Havana Field Station

704 North Schrader Avenue

Havana, Illinois 62644-1055

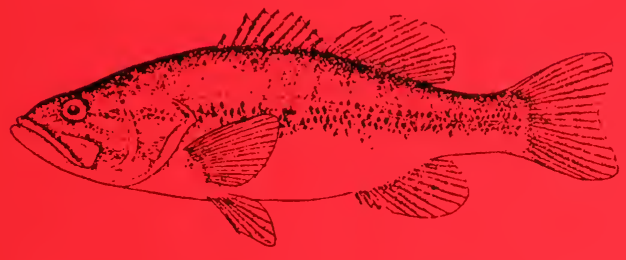

November 1999

Center for Aquatic Ecology Technical Report 99/15 



\title{
The Long-term Illinois River Fish Population Monitoring Program
}

\author{
F-101-R
}

Final Report

Todd M. Koel and Richard E. Sparks

Illinois Natural History Survey

LTRMP Havana Field Station

704 North Schrader Avenue

Havana, Illinois 62644-1055

November 1999
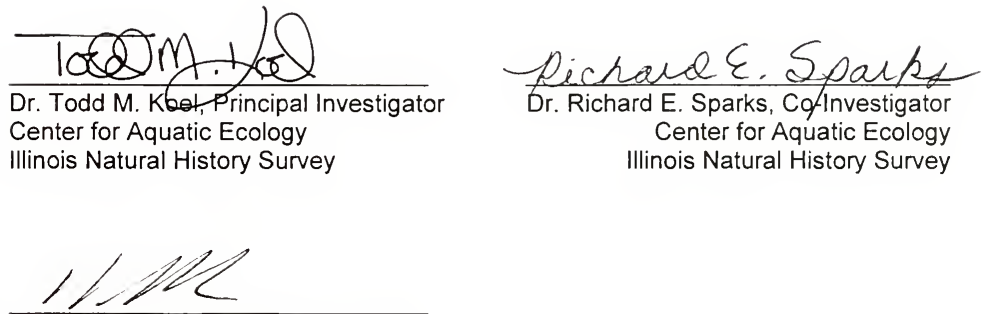

Dr. Daniel Soluk, Director

Center for Aquatic Ecology

Illinois Natural History Survey 



\title{
The Long-term Illinois River Fish Population Monitoring Program
}

\author{
F-101-R \\ Segments 6-10 \\ Final Report
}

\begin{abstract}
to be submitted to the
Illinois Department of Natural Resources

and

U.S. Fish and Wildlife Service
\end{abstract}

Todd M. Koel and Richard E. Sparks

Illinois Natural History Survey

LTRMP Havana Field Station

704 North Schrader Avenue

Havana, Illinois 62644-1055

November 1999 



\section{DISCLAIMER}

The findings, conclusions, and views expressed herein are those of the

researchers and should not be considered as the official position of the United States Fish and Wildlife Service or the Illinois Department of Natural Resources.

\section{ACKNOWLEDGMENT OF SUPPORT}

The Long-term Illinois River Fish Population Monitoring Program (F-101-R) is supported by the Federal Aid in Sport Fish Restoration Act (P.L. 81-681, DingellJohnson/Wallop-Breaux). 



\section{EXECUTIVE SUMMARY}

During late August and September each year 1994-1998, we sampled 26 sites on the Illinois River Waterway and one site on Reach 26 of the Mississippi River by electrofishing to monitor fish communities. From 1994-1998, we collected a total of 25,921 fish representing 62 species (plus five hybrids) from fourteen families during 125.70 hours of sampling at 26 sites on the Illinois Waterway and a single site on the Mississippi River. Of these fishes, 25,278 individuals were collected from the Illinois Waterway sites, and 643 were collected from Brickhouse Slough of the Mississippi River. The year with the greatest overall catch of fishes was 1995 (7941 individuals, CPUE $_{N}=325$ fish per hour) and the year with the lowest overall catch of fishes was 1994 (3421 individuals, CPUE $_{N}=131$ fish per hour). For all stations combined, the greatest number of species were collected in 1995 (48 species plus 3 hybrids) and the least were in 1997 ( 38 species plus 4 hybrids). The number of species collected from upper waterway reaches ranged from 12 for Starved Rock in 1996 to 24 for Marseilles in 1995. The number of species collected from middle river reaches ranged from 23 for La Grange Reach in 1997 to 34 for Peoria Reach in 1996. The number of species collected from the lower river (Alton Reach) ranged from 18 in 1994 to 25 in 1995 . The Peoria Reach consistently had highest species richness during all years (1994-1998) of sampling for this project. 



\section{TABLE OF CONTENTS}

Title and Signature Page

DISCLAIMER

ACKNOWLEDGMENT OF SUPPORT $\ldots \ldots \ldots \ldots \ldots \ldots \ldots \ldots$ ii

EXECUTIVE SUMMARY $\ldots \ldots \ldots \ldots \ldots \ldots \ldots \ldots \ldots \ldots \ldots \ldots \ldots \ldots$

TABLE OF CONTENTS $\ldots \ldots \ldots \ldots \ldots \ldots \ldots \ldots \ldots \ldots \ldots \ldots \ldots \ldots \ldots \ldots$

LIST OF TABLES $\ldots \ldots \ldots \ldots \ldots \ldots \ldots \ldots \ldots \ldots \ldots \ldots \ldots \ldots$

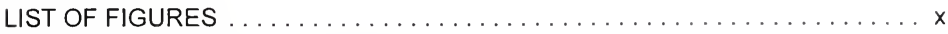

INDEX TO JOB ACCOMPLISHMENTS ................. .

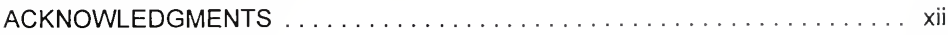

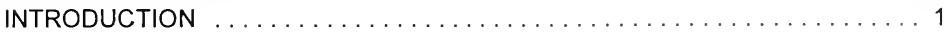

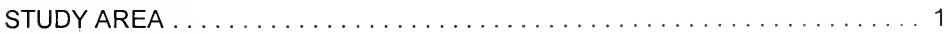

MATERIALS AND METHODS $\ldots \ldots \ldots \ldots \ldots \ldots \ldots \ldots \ldots \ldots \ldots$

A. Criteria for Sampling $\ldots \ldots \ldots \ldots \ldots \ldots \ldots \ldots \ldots \ldots$

B. Data Analysis . . . . . . . . . . . . . . . . . . . . 10

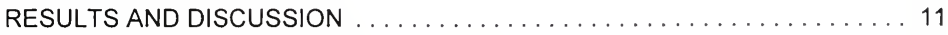

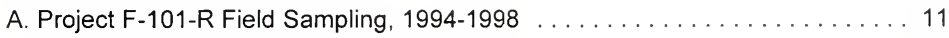

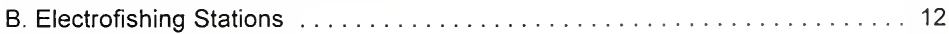

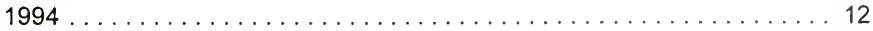

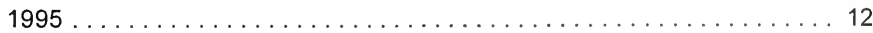

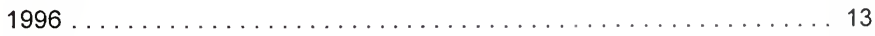

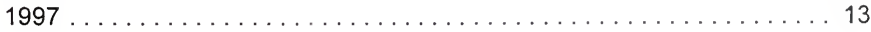



1998

C. Catch Rates in Numbers of Individuals Collected per Hour 14

Numbers of Fish Collected 14 Rankings by Relative Abundance 25 CPUE $_{N}$ of Five Most Numerically Abundant Species 26

D. Catch Rates in Weights (pounds) Collected per Hour 32

E. Details of 1998 Sampling 44 CONCLUSIONS 44

LITERATURE CITED 48 APPENDIX A 50 APPENDIX B 52

APPENDIX C 53

APPENDIX D 54

APPENDIX E 55

APPENDIX F 56 APPENDIX G 57 



\section{LIST OF TABLES}

Table 1. Station information and characteristics during sampling in 1994

Table 2. Station information and characteristics during sampling in 1995

Table 3. Station information and characteristics during sampling in 1996

Table 4. Station information and characteristics during sampling in 1997

Table 5. Station information and characteristics during sampling in 1998

Table 6. Numbers of individuals of each fish species collected per hour of electrofishing on Reach 26 of the Mississippi River (Brickhouse Slough) and on six reaches of the Illinois River Waterway in 1994

Table 7. Species ranked by relative abundance in number of fish collected per hour for 1994 16

Table 8. Numbers of individuals of each fish species collected per hour of electrofishing on Reach 26 of the Mississippi River (Brickhouse Slough) and on six reaches of the Illinois River Waterway in 1995

Table 9. Species ranked by relative abundance in number of fish collected per hour for 1995 

Table 10. Numbers of individuals of each fish species collected per hour of electrofishing on Reach 26 of the Mississippi River (Brickhouse Slough) and on six reaches of the Illinois River Waterway in 1996

Table 11. Species ranked by relative abundance in number of fish collected per hour for 1996

Table 12. Numbers of individuals of each fish species collected per hour of electrofishing on Reach 26 of the Mississippi River (Brickhouse Slough) and on six reaches of the Illinois River Waterway in 1997

Table 13. Species ranked by relative abundance in number of fish collected per hour for 1997

Table 14. Numbers of individuals of each fish species collected per hour of electrofishing on Reach 26 of the Mississippi River (Brickhouse Slough) and on six reaches of the Illinois River Waterway in 1998

Table 15. Species ranked by relative abundance in number of fish collected per hour for 1998

Table 16. Pounds of each fish species collected per hour of electrofishing on Reach 26 of the Mississippi River (Brickhouse Slough) and on six reaches of the Illinois River Waterway in 1994 

Table 17. Species ranked by relative abundance in pounds of fish collected per hour for 1994

Table 18. Pounds of each fish species collected per hour of electrofishing on Reach 26 of the Mississippi River (Brickhouse Slough) and on six reaches of the Illinois River Waterway in 1995 .

Table 19. Species ranked by relative abundance in pounds of fish collected per hour for 1995 36

Table 20. Pounds of each fish species collected per hour of electrofishing on Reach 26 of the Mississippi River (Brickhouse Slough) and on six reaches of the Illinois River Waterway in 1996

Table 21. Species ranked by relative abundance in pounds of fish collected per hour for 1996 38

Table 22. Pounds of each fish species collected per hour of electrofishing on Reach 26 of the Mississippi River (Brickhouse Slough) and on six reaches of the Illinois River Waterway in 1997

Table 23. Species ranked by relative abundance in pounds of fish collected per hour for 1997 

Table 24. Pounds of each fish species collected per hour of electrofishing on Reach 26 of the Mississippi River (Brickhouse Slough) and on six reaches of the Illinois River Waterway in 1998

Table 25. Species ranked by relative abundance in pounds of fish collected per hour for 1998 . 



\section{LIST OF FIGURES}

Figure 1. Three segments of the Illinois River sampled by electrofishing to monitor fish communities in $1997 \ldots \ldots \ldots . \ldots 3$

Figure 2. Catch per hour of gizzard shad from 1994 through 1998 in the lower, middle, and upper Illinois River waterway . . . . . . . 27

Figure 3. Catch per hour of common carp from 1994 through 1998 in the lower, middle, and upper Illinois River waterway . . . . . . . . 28

Figure 4. Catch per hour of emerald shiner from 1994 through 1998 in the lower, middle, and upper Illinois River waterway . . . . . . . . 29

Figure 5. Catch per hour of bluegill from 1994 through 1998 in the lower, middle, and upper Illinois River waterway 30

Figure 6. Catch per hour of freshwater drum from 1994 through 1998 in the lower, middle, and upper Illinois River waterway 



\section{INDEX TO JOB ACCOMPLISHMENTS}

Job $1^{\text {a }} \quad$ Prepare electrofishing equipment and train staff

Job 2 Sample by electrofishing at 27 sites along the Illinois

River Waterway and Reach 26 of the Mississippi River

Job 3

Update computer database

Job 4

Analyze data

Job 5

Presentation of results

a Job numbers and titles refer to the F-101-R annual work plans 


\section{ACKNOWLEDGMENTS}

Project F-101-R is supported by the Federal Aid to Sportfish Restoration Act (P.L. 81-681, Dingell-Johnson/Wallop-Breaux), with funds administered by the U.S. Fish and Wildlife Service and the Illinois Department of Natural Resources (IDNR). Mr. Larry Dunham (IDNR); Mr. Bill Bertrand (IDNR); Mr. Michael Sweet (IDNR); Dr. David Thomas, Chief of the Illinois Natural History Survey (INHS); Dr. Edward Armbrust, Acting Chief (INHS); and Dr. David Philipp, former Director of the Center for Aquatic Ecology (INHS); provided administrative support. Ms. Cammy Smith of the Long Term Resource Monitoring Program Field Station at Havana provided secretarial support and data entry and verification. Mr. Thad Cook, Mr. Kip Stevenson, and Mr. Scott Whitney assisted with the field work. Mr. K. Douglas Blodgett was Co-Investigator of this project until 1998. Mr. Thomas Lerczak was Project Manager until 1995. This survey was originally conceived and initiated in 1957 by the late Dr. William C. Starrett. 



\section{INTRODUCTION}

The goals of the Long-term Illinois River Fish Population Monitoring Program include: 1) determination of spatial and temporal trends in fish populations of the Illinois River; 2) Develop a long-term fisheries database useful for evaluating resource management strategies; and 3) provide information required to manage the fishery of the Illinois River. This report presents a summary of data collected 19941998 during segments 6-10 of federal aid project F-101-R, The Long-term Illinois River Fish Population Monitoring Program. Previous summaries of the long-term data set, begun in 1957, were given by Sparks and Starrett (1975), Sparks (1977), Sparks and Lerczak (1993), Lerczak and Sparks (1994), and Lerczak et al. (1994). The annual reports for project F-101-R have continuously built on previously collected data. The format used in this report is patterned after previous annual reports of this project (Lerczak et al. 1993, 1994, 1995, and 1996 and Koel et al. 1997 and 1998) to allow for easy comparisons of data among years. The objective of this report is to provide a summary document of Illinois River fish population data collected 1994-1998 during federal aid project F-101-R.

\section{STUDY AREA}

Twenty-six fish sampling sites were at fixed locations along the Illinois Waterway as defined by Sparks and Starrett (1975:347) and Lerczak et al. (1994:9) 
(Table 1). Twenty-four of the sites were along the Illinois River, with two additional sites on the lower Des Plaines River, which along with the Illinois River is part of the Illinois Waterway. One additional site was on the Mississippi River (Figure 1).

Seventeen of the sites were in side channels; the rest of the sites were in other habitats, including the main channel border, or in a combination of habitat types (see Lerczak et al. 1994:9). By calculating the average river mile of each fish sampling site for the total period of record (1957-present), the sites were "renamed" in 1998 to reflect river mile (Figure 1). For this and all subsequent reports, we will refer to sites by these approximate average river miles (site mile, Tables 1-5) for use in all figures and tables. In text we will refer to sites by average river mile as well as by common site descriptions (e.g., Brickhouse Slough, Mortland Island, etc.).

\section{MATERIALS AND METHODS}

Fish populations were sampled by electrofishing from a 16-ft (5-m) aluminum boat using a Homelite 3000-watt, three-phase AC electric generator. Boat configuration includes three poles extended from the bow with metal electrodes, connecting to the electric generator, extended from the ends of the poles to approximately 20 inches $(0.5 \mathrm{~m})$ below the water line. The same generator and electrode configuration have been used since 1957.

Prior to fish sampling, water quality and flow measurements (e.g., dissolved 


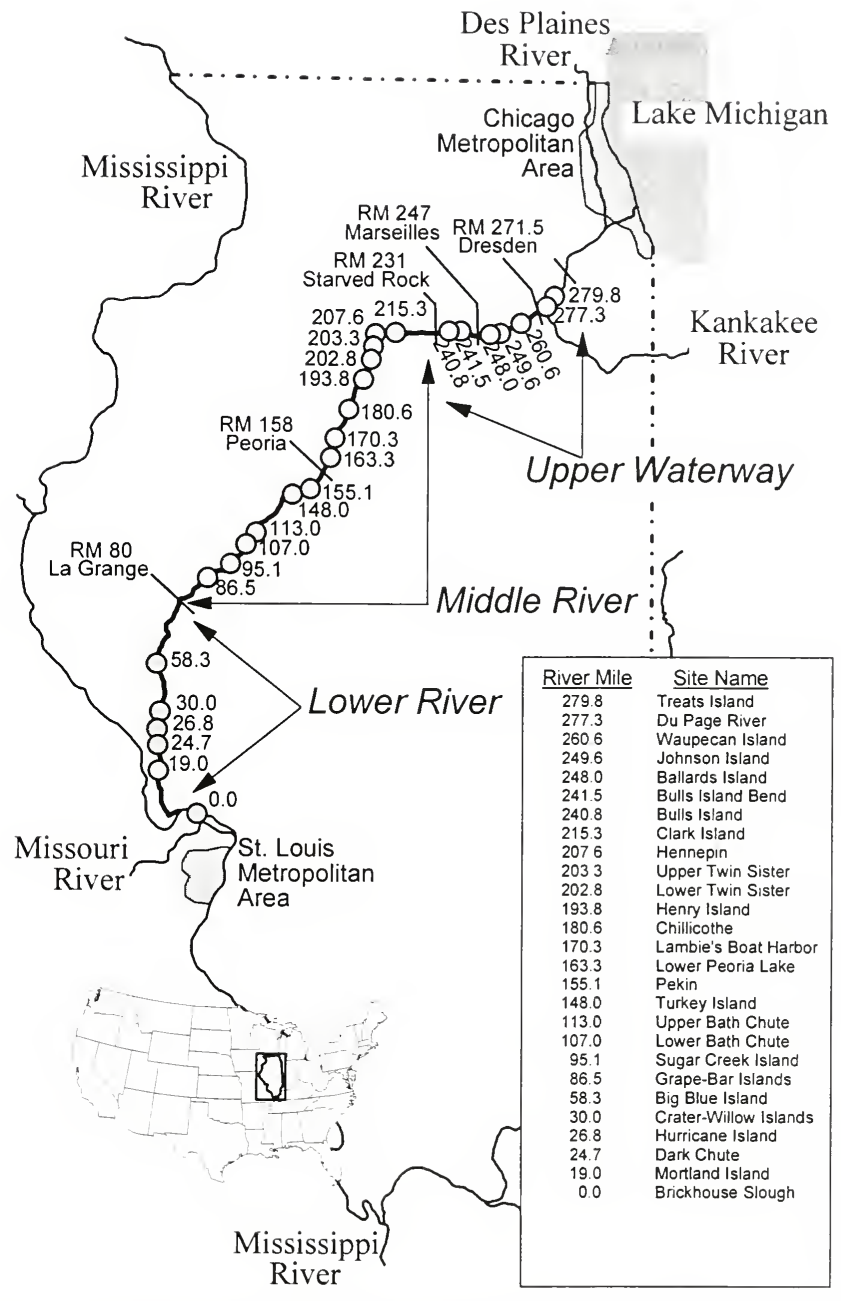

Figure 1. Three segments of the Illinois River Waterway sampled by electrofishing to monitor fish communities during August and September, 1994-1998. 


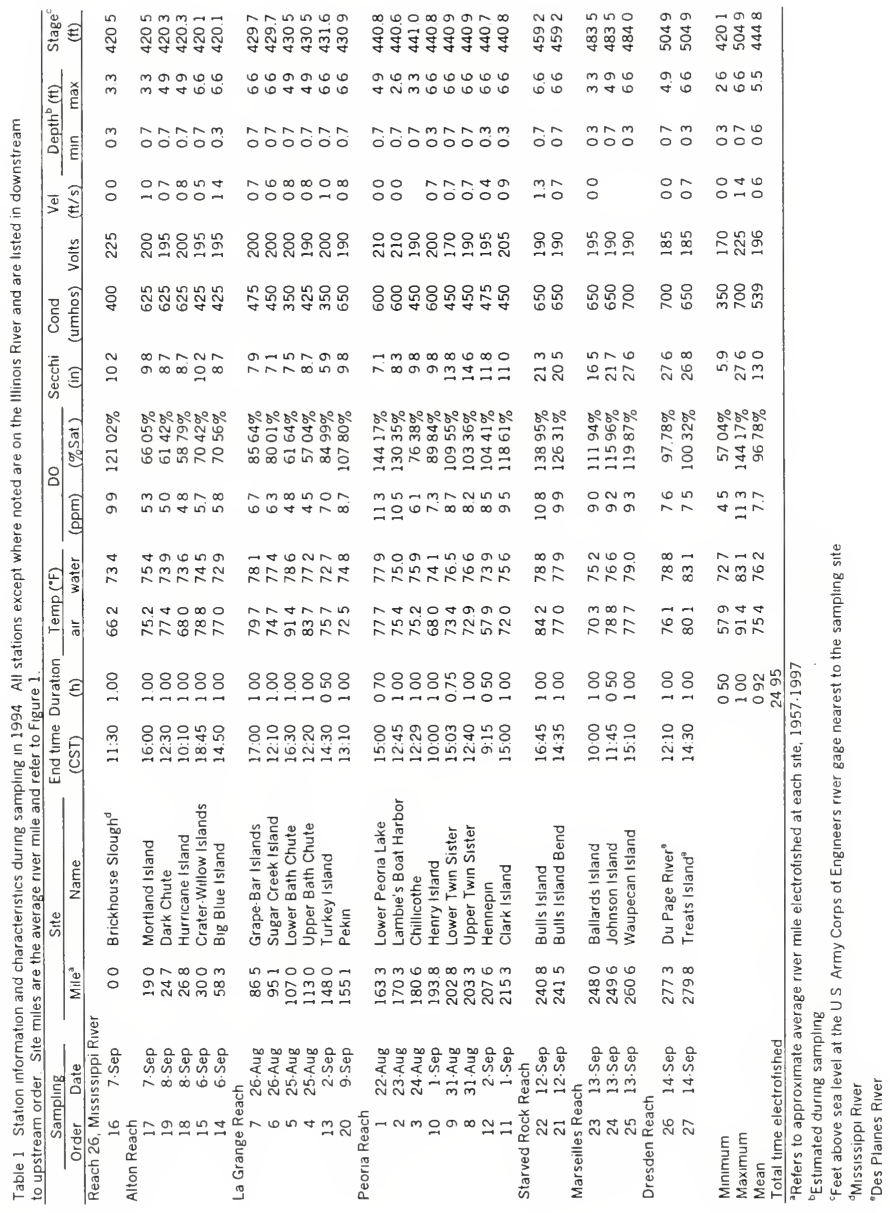




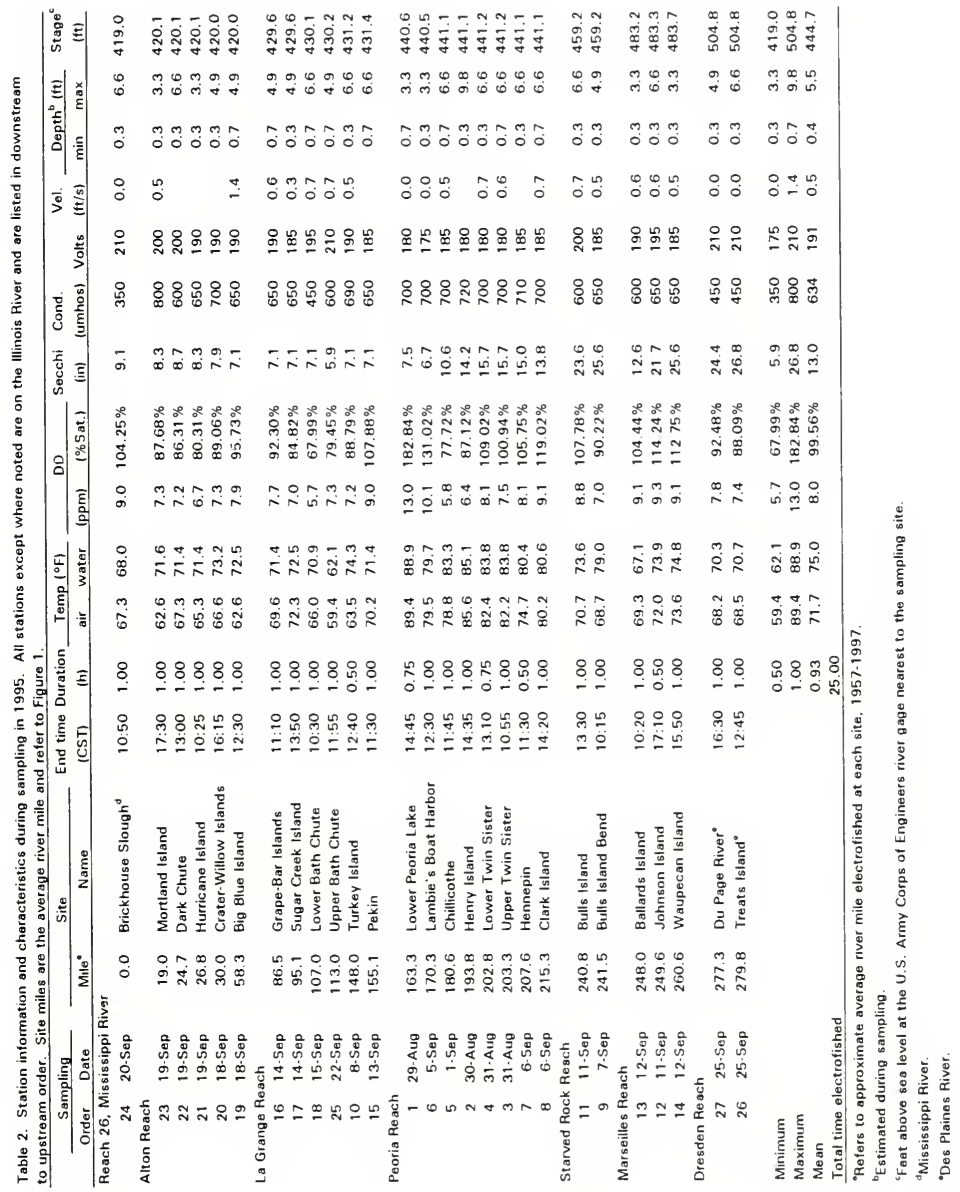




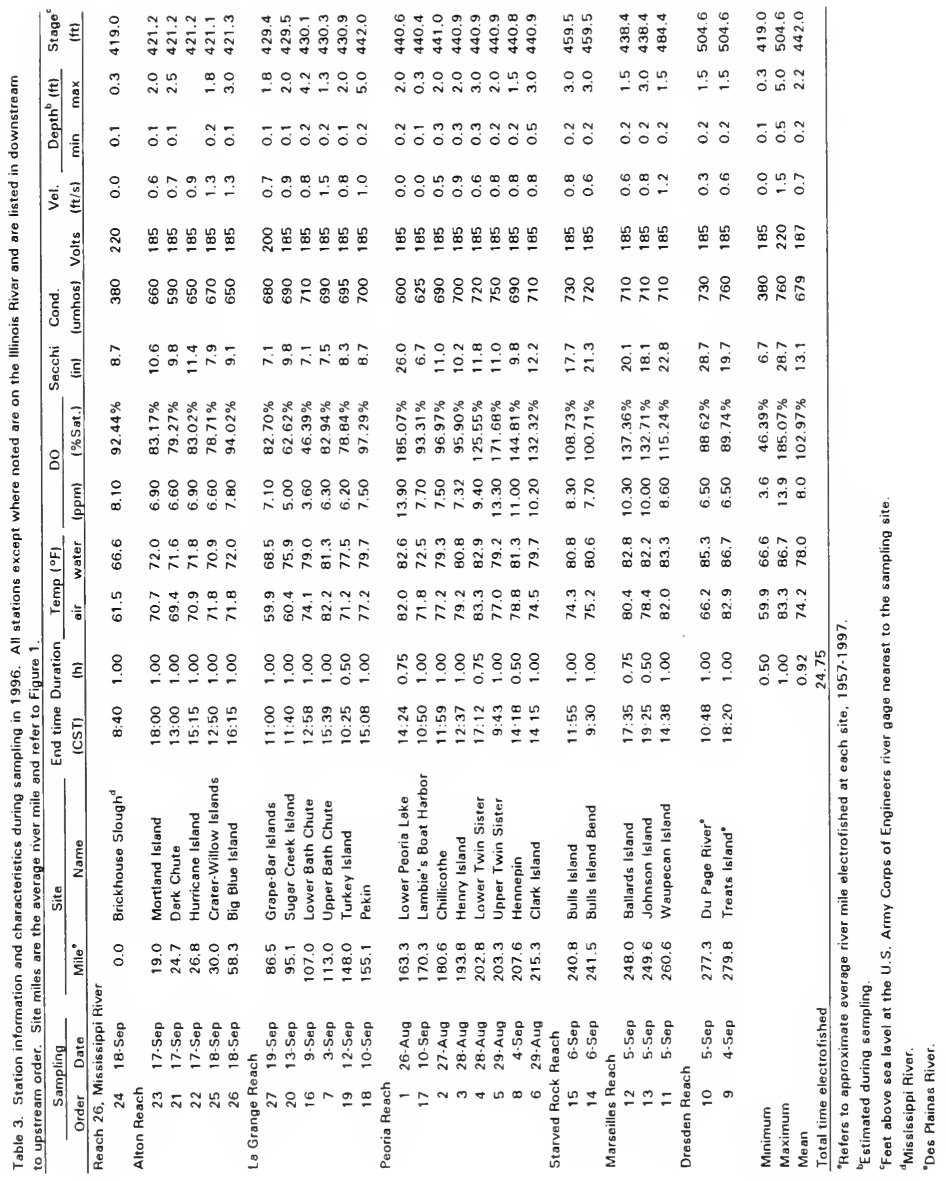




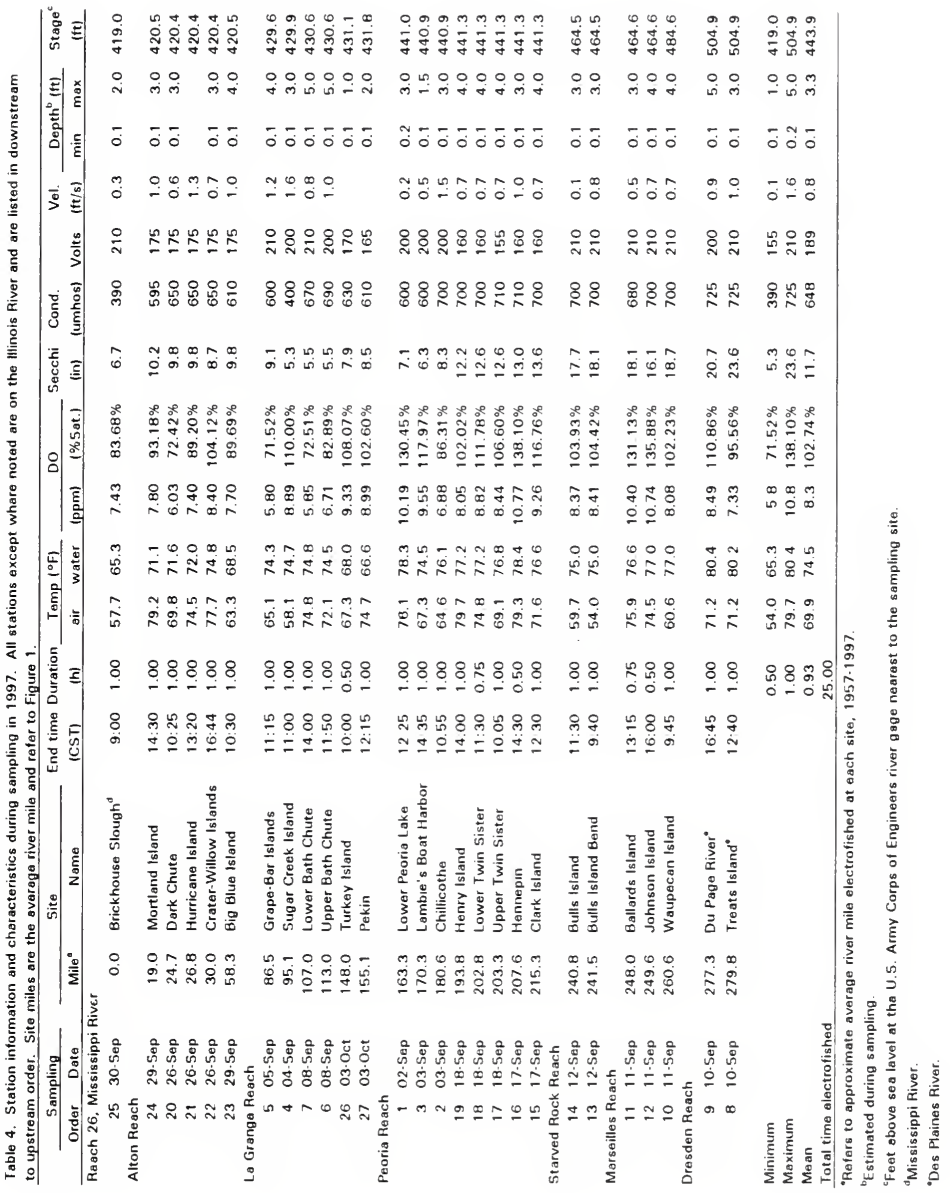




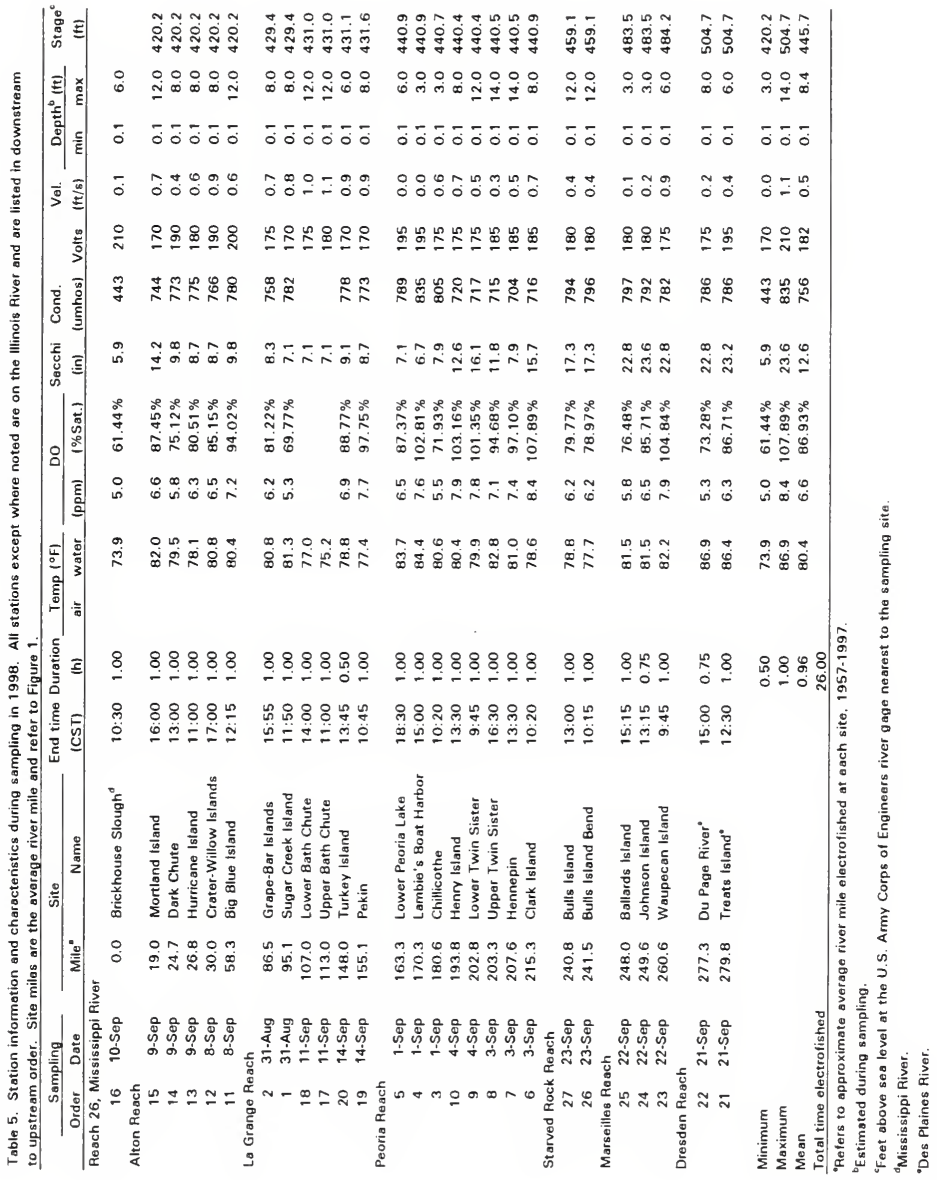


oxygen, specific conductance, temperature, surface velocity) were taken at the upstream end of each site. Sampling at each site typically lasted one hour, with all obvious structure (e.g. downed trees, woody debris, rock rip-rap) intensively sampled for fishes. Stunned fish were gathered with a dip net $(1 / 4-$ in $[0.64-\mathrm{cm}]$ mesh) and stored in an oxygenated livewell until sampling was completed. Fish were then identified to species, measured (total length and weight), inspected for externally visible abnormalities (sores, fin erosion, etc.), and returned to the water. A detailed description of the electrofishing method and equipment are provided by Lerczak et al. (1994, Appendix A).

\section{A. Criteria for Sampling}

Fish collection by the Long-term Illinois River Fish Population Monitoring Program occurs during the last week in August and September each year to increase the probability of collecting young-of-year fishes with a $1 / 4$ inch mesh dip net. Sampling at each site is only conducted if river water levels are low and stable, as determined by the U.S. Army Corps of Engineers, who maintain gage sites along the length of the Illinois River waterway. Also, sampling for this project does not occur if water temperatures have fallen unseasonably low (below $58 \mathrm{C}$ ), which are not typically reached on the Illinois River until mid-October (Kofoid 1903, LTRMP unpublished data 1999). 


\section{B. Data Analysis}

At each site, number of individual fish and total weight (pounds) were tallied for each species. Fish catch rates were calculated as the number of individuals collected per hour of electrofishing $\left(C P \cup E_{N}\right)$ and as weight in pounds collected per hour of electrofishing (CPUE $E_{W}$. For 1994-1998, data from sites were grouped into reaches defined by navigation dams (Figure 1 ) as follows: Alton Reach, river mile (RM) 0-80; La Grange Reach, RM 80-158; Peoria Reach, RM 158-231; Starved Rock Reach, RM 231-247; Marseilles Reach, RM 247-271.5; and Dresden Reach, RM 271.5-286 on the Des Plaines River. Data from reaches were combined further into three groups (lower and middle Illinois River segments, and the upper Illinois Waterway segment) defined by their location along the river and by the amount of off-channel habitat accessible to fish per unit length of river (Lerczak et al. 1994:5 and Figure 1). Lerczak et al. (1994, 1995, and 1996) showed that river fish communities of the three segments differed substantially enough to give segment designations biological meaning. Separate tables were constructed listing only those species that accounted for at least $95 \%$ of the total catch rates. This percentage was arbitrarily chosen to emphasize species of most importance in analyzing fish community composition. 


\section{RESULTS AND DISCUSSION (Job 4)}

\section{A. Project F-101-R Field Sampling, 1994-1998}

Before each fish sampling season began, all equipment was tested and repaired as necessary. Due to the arrival of the new project manager Koel, and loss of all senior staff from the Havana Field Station during this project, training for new staff was more intensive than that needed in recent years; new staff were trained in electrofishing methods and safety procedures (Job 1). All field sampling for this project occurred during late August and September each year, with typically one hour spent electrofishing at each of 27 fixed sites, each year (Job 2).

The long-term database was converted from R-Base computer format to an updated, Microsoft Access 97 format. Data collected during F-101-R were entered directly into this database, and verified against original field data sheets until no errors were detected (Job 3). The original data sheets of this project (1957-1997) were originally stored in a vault along Quiver Creek at Forbes Biological Station. Due to moisture problems and a fire hazard within this vault, the data were moved and are now stored in flame-resistant cabinets at the Long Term Resource Monitoring Program Field Station at 704 N. Schrader Avenue, Havana (Job 3). Data analysis has occurred at the LTRMP Havana Field Station (Job 4) with results presented at several public and scientific meetings. 


\section{B. Electrofishing Stations}

All 27 long-term sites were sampled for fishes and physio-chemical parameters each year from 1994-1997. Site listings and water quality parameters are provided by Tables 1-5 (Job 5). All values were within the ranges expected based upon previous sampling (see Lerczak et al. 1994:17-24). All sites were sampled with water temperatures and river levels (Tables 1-5) within our previously established criteria.

1994. All stations were sampled between 22 August and 29 September 1994, taking 27.95 hours, with sampling times ranging from 0.5 to 1.0 hour (Table 1 ). Sampling was conducted in full daylight between the hours of 8:45 AM and 6:45 PM. The ranges for physical measurements during the 1994 sampling season were as follows: air temperature, 57.9-91.4 F; water temperature, 72.7-83.1 F; dissolved oxygen concentration, 4.5-11.3 ppm; Secchi disk transparency, 5.9-27.6 inches; specific conductance, 350-700 umhos; surface velocity, 0.0-1.4 ft/s; water depth, 0.3$6.6 \mathrm{ft}$ (Table 1).

1995. All stations were sampled between 29 August and 25 September 1995, taking 25.00 hours, with sampling times ranging from 0.5 to 1.0 hour (Table 2 ). Sampling was conducted in full daylight between the hours of 9:15 AM and 5:30 PM. The ranges for physical measurements during the 1995 sampling season were as 
follows: air temperature, 59.4-89.4 F; water temperature, 62.1-88.9 F; dissolved oxygen concentration, 5.7-13.0 ppm; Secchi disk transparency, 5.9-26.8 inches; specific conductance, 350-800 umhos; surface velocity, 0.0-1.4 ft/s; water depth, 0.3$9.8 \mathrm{ft}$ (Table 2).

1996. All stations were sampled between 26 August and 19 September 1996, taking 24.75 hours, with sampling times ranging from 0.5 to 1.0 hour (Table 3 ). Sampling was conducted in full daylight between the hours of 7:40 AM and 7:25 PM. The ranges for physical measurements during the 1996 sampling season were as follows: air temperature, 59.9-83.3 F; water temperature, 66.6-86.7 F; dissolved oxygen concentration, 3.6-13.9 ppm; Secchi disk transparency, 6.7-28.7 inches; specific conductance, 380-760 umhos; surface velocity, 0.0-1.5 ft/s; water depth, 0.1$5.0 \mathrm{ft}$ (Table 3).

1997. All stations were sampled between 2 September and 3 October 1997 , taking 25.00 hours, with sampling times ranging from 0.5 to 1.0 hour (Table 4). Sampling was conducted in full daylight between the hours of 8:00 AM and 4:45 PM. The ranges for physical measurements during the 1997 sampling season were as follows: air temperature, 54.0-79.7 F; water temperature, 65.3-80.4 F; dissolved oxygen concentration, 5.8-10.8 ppm; Secchi disk transparency, 5.3-23.6 inches; specific conductance, 390-725 umhos; surface velocity, 0.1-1.6 ft/s; water depth, 0.1$5.0 \mathrm{ft}$ (Table 4). 
1998. All stations were sampled between 31 August and 23 September 1998, taking 26.00 hours, with sampling times ranging from 0.5 to 1.0 hour (Table 5). Sampling was conducted in full daylight between the hours of 8:45 AM and 6:30 PM. The ranges for physical measurements during the 1998 sampling season were as follows: air temperature, (not measured); water temperature, 73.9-86.9 F; dissolved oxygen concentration, 5.0-8.4 ppm; Secchi disk transparency, 5.9-23.6 inches; specific conductance, 443-835 umhos; surface velocity, $0.0-1.1 \mathrm{ft} / \mathrm{s}$; water depth, 0.0$14.0 \mathrm{ft}$ (Table 5).

\section{Catch Rates in Numbers of Individuals}

In this report, for each year (1994-1998) catch rates of the number of individuals collected per hour of electrofishing are calculated for each of the seven Illinois River navigation reaches (Figure 1). Similar summaries are presented for fish weights. Common names used throughout this report follow Robins et al. (1991). Common and scientific names are listed in APPENDIX A. Number of individuals of each fish species collected per hour of electrofishing and species rankings by waterway reach and year are summarized by Tables 6 through 15 .

Numbers of Fish Collected. From 1994-1998, we collected a total of 25,921 fish representing 62 species (plus five hybrids) from fourteen families during 125.70 hours of sampling at 26 sites on the Illinois Waterway and a single site on the 
Table 6 Number of individuais of each fish species collected per hour of electrofishing (CPUE $)$ at Reach 26 of the Mississippi

River (Brickhouse Slough) and at six reaches of the Illinois River Waterway in 1994

\begin{tabular}{|c|c|c|c|c|c|c|c|c|}
\hline \multirow{4}{*}{ Species } & \multirow{2}{*}{\multicolumn{7}{|c|}{$\begin{array}{l}\text { Reach and Hours Fished } \\
\text { Starved }\end{array}$}} & \multirow{4}{*}{$\begin{array}{r}\text { Overall } \\
\text { CPUE }_{\mathrm{N}} \\
2695 \\
\end{array}$} \\
\hline & & & & & & & & \\
\hline & \multirow{2}{*}{$\begin{array}{c}\text { Reach } 26 \\
100\end{array}$} & \multirow{2}{*}{$\begin{array}{l}\text { Alton } \\
500\end{array}$} & \multirow{2}{*}{$\begin{array}{l}\text { La Grange } \\
\quad 850\end{array}$} & \multirow{2}{*}{$\begin{array}{l}\text { Peoria } \\
695\end{array}$} & Rock & Marseilles & \multirow{2}{*}{$\begin{array}{c}\text { Dresden } \\
200 \\
\end{array}$} & \\
\hline & & & & & 200 & 250 & & \\
\hline \multicolumn{9}{|l|}{ Lepisosteidae } \\
\hline longnose gar & & & 012 & & & & & 004 \\
\hline \multicolumn{9}{|l|}{ Amidae } \\
\hline bowfin & & 020 & & & & & & 004 \\
\hline \multicolumn{9}{|l|}{ Clupeidae } \\
\hline gizzard shad & 7.00 & 1240 & 1071 & 647 & 7.00 & 1480 & 2250 & 1117 \\
\hline skipjack herring & & & & 086 & & 0.40 & & 026 \\
\hline threadfin shad & & 040 & & 633 & & & & 171 \\
\hline \multicolumn{9}{|l|}{ Hiodontıdae } \\
\hline goldeye & & & 024 & 029 & & & & 015 \\
\hline Cyprınidae & & & & & & & & \\
\hline bullhead minnow & & & & & 1.50 & 2680 & 6850 & 1.19 \\
\hline bluntnose minnow & & & 012 & 058 & 800 & 120 & 400 & 768 \\
\hline common carp & 3.00 & 680 & 2412 & 691 & 400 & 360 & 9.50 & 12.10 \\
\hline common carp $\times$ goldfish & & & & & & 0.80 & 350 & 033 \\
\hline emerald shiner & 400 & 180 & 165 & 1856 & 2100 & 3120 & 1000 & 1098 \\
\hline golden shıner & & & & & & 080 & 2.50 & 026 \\
\hline goldfish & & & 0.12 & 043 & & & 050 & 019 \\
\hline minnow (unid.) & & & & 029 & & 040 & & 011 \\
\hline red shiner & & & 1.29 & & 100 & 240 & & 071 \\
\hline sand shiner & & & & & 400 & 680 & & 093 \\
\hline silver chub & 100 & & & 1.01 & & & & 030 \\
\hline spottail shiner & & & & 043 & & 120 & 15.50 & 137 \\
\hline Catostomidae & & & & & & & & \\
\hline bıgmouth buffalo & & 200 & 624 & 417 & & & & 341 \\
\hline golden redhorse & & & 012 & 043 & 050 & 1.60 & 1.00 & 041 \\
\hline highfin carpsucker & & & & & 050 & & & 004 \\
\hline northem hog sucker & & & & & & 040 & & 004 \\
\hline quillback & & & & 014 & & & & 0.04 \\
\hline river carpsucker & & & 012 & 302 & 050 & 160 & 050 & 104 \\
\hline shorthead redhorse & & & 188 & 245 & & & 100 & 1.30 \\
\hline smailmouth buffalo & 4.00 & 360 & 482 & 5.90 & 900 & 1.60 & & 468 \\
\hline Ictaluridae & & & & & & & & \\
\hline channel catfish & 100 & 1620 & 494 & 173 & 450 & 1.20 & & 549 \\
\hline flathead cattish & & 020 & 047 & 014 & & & 0.50 & 026 \\
\hline yellow bullhead & & 040 & & & & & & 007 \\
\hline Atherinidae & & & & & & & & \\
\hline brook silversıde & & & & & & & 050 & 0.04 \\
\hline Percichthyidae & & & & & & & & \\
\hline white bass & 2.00 & 2.60 & 1035 & 518 & 350 & 240 & & 564 \\
\hline Centrarchidae & & & & & & & & \\
\hline black crappie & 1200 & 920 & 11.41 & 216 & & & 100 & 638 \\
\hline bluegill & 33.00 & 4460 & 30.71 & 31.94 & 350 & 960 & 1000 & 2931 \\
\hline bluegill $\times$ green sunfish & & & & 0.58 & & & 050 & 019 \\
\hline green sunfish & 1.00 & 080 & 059 & 1295 & 1.00 & 1.60 & 1450 & 5.01 \\
\hline largemouth bass & 12.00 & 7.40 & 435 & 518 & 050 & 760 & 1.00 & 534 \\
\hline orangespotted sunfish & 2.00 & & 0.12 & 0.14 & 0.50 & & 050 & 022 \\
\hline pumpkinseed & & & & & & & 0.50 & 0.04 \\
\hline redear sunfish & 1.00 & & & & & & & 0.04 \\
\hline rock bass & & & & & & & 1.00 & 004 \\
\hline smallmouth bass & & & 0.12 & 043 & 250 & 240 & 500 & 093 \\
\hline warmouth & & 060 & 035 & & & & & 022 \\
\hline white crappie & & & 0.94 & 0.29 & & & & 037 \\
\hline Percidae & & & & & & & & \\
\hline sauger & 1.00 & 0.20 & 071 & 0.72 & & & & 048 \\
\hline slenderhead darter & & & & & & 040 & & 004 \\
\hline Sciaenidae & & & & & & & & \\
\hline freshwater drum & 27.00 & 1240 & 1282 & 1122 & & 2.00 & 050 & 1046 \\
\hline Total number per hour & 111.00 & 12180 & 12941 & 130.94 & 7300 & 12280 & 17450 & 131.05 \\
\hline Number of species/hybrids & $15 / 0$ & $18 / 0$ & $26 / 0$ & $28 / 1$ & $18 / 0$ & $22 / 1$ & $22 / 2$ & $42 / 2$ \\
\hline
\end{tabular}


Table 7. Species ranked by relative abundance in number of fish collected per hour for 1994 . Species were added to the list in descending order of abundance until $95 \%$ of the total catch for that reach was obtained. Percentages are in parentheses.

\begin{tabular}{|c|c|c|c|c|c|c|}
\hline \multirow[b]{2}{*}{ Species } & \multicolumn{6}{|c|}{ Rankings by Reach } \\
\hline & Alton & La Grange & Peoria & $\begin{array}{l}\text { Starved } \\
\text { Rock }\end{array}$ & Marseilles & Dresden \\
\hline \multicolumn{7}{|l|}{ Clupeidae } \\
\hline gizzard shad & $3(10.2)$ & $5(8.3)$ & $6(4.9)$ & $4(9.6)$ & $3(12.1)$ & $2(12.9)$ \\
\hline threadfin shad & & & $7(4.8)$ & & & \\
\hline \multicolumn{7}{|l|}{ Cyprinidae } \\
\hline bluntnose minnow & & & & $9(2.1)$ & $2(21.8)$ & $1(39.3)$ \\
\hline bullhead minnow & & & & $3(11.0)$ & & $8(2.3)$ \\
\hline common carp & $6(5.6)$ & $2(18.6)$ & $5(5.3)$ & $6(5.5)$ & $7(2.9)$ & $6(5.4)$ \\
\hline common carp $\times$ goldfish & & & & & & $9(2.0)$ \\
\hline emerald shiner & & $12(1.3)$ & $2(14.2)$ & $1(28.8)$ & $1(25.4)$ & $5(5.7)$ \\
\hline golden shiner & & & & & & $10(1.4)$ \\
\hline red shiner & & & & $10(1.4)$ & $8(2.0)$ & \\
\hline sand shiner & & & & $6(5.5)$ & $6(5.5)$ & \\
\hline silver chub & & & $15(0.8)$ & & & \\
\hline spottail shiner & & & & & & $3(8.9)$ \\
\hline \multicolumn{7}{|l|}{ Catostomidae } \\
\hline bigmouth buffalo & $9(1.6)$ & $7(4.8)$ & $10(3.2)$ & & & \\
\hline golden redhorse & & & & & $10(1.3)$ & \\
\hline river carpsucker & & & $11(2.3)$ & & $10(1.3)$ & \\
\hline shorthead redhorse & & $11(1.5)$ & $12(1.9)$ & & & \\
\hline smallmouth buffalo & $7(3.0)$ & $9(3.7)$ & $8(4.5)$ & $2(12.3)$ & $10(1.3)$ & \\
\hline \multicolumn{7}{|l|}{ Ictaluridae } \\
\hline channel catfish & $2(13.3)$ & $8(3.8)$ & $14(1.3)$ & $5(6.2)$ & $11(0.6)$ & \\
\hline \multicolumn{7}{|l|}{ Percichthyidae } \\
\hline white bass & $8(2.1)$ & $6(8.0)$ & $9(4.0)$ & $7(4.8)$ & $8(2.0)$ & \\
\hline \multicolumn{7}{|l|}{ Centrarchidae } \\
\hline black crappie & $4(7.6)$ & $4(8.8)$ & $13(1.6)$ & & & $11(1.0)$ \\
\hline bluegill & $1(36.6)$ & $1(23.7)$ & $1(24.4)$ & $7(4.8)$ & $4(7.8)$ & $5(5.7)$ \\
\hline green sunfish & & & $3(9.9)$ & & $10(1.3)$ & $4(8.3)$ \\
\hline largemouth bass & $5(6.1)$ & $10(3.4)$ & $9(4.0)$ & & $5(6.2)$ & \\
\hline smallmouth bass & & & & $8(3.4)$ & $8(2.0)$ & $7(2.9)$ \\
\hline \multicolumn{7}{|l|}{ Sciaenidae } \\
\hline freshwater drum & $3(10.2)$ & $3(9.9)$ & $4(8.6)$ & & $9(1.6)$ & \\
\hline \multicolumn{7}{|l|}{ Numbers of fishes } \\
\hline accounting for $95 \%$ & 10 & 12 & 16 & 12 & 16 & 12 \\
\hline
\end{tabular}


Table 8 Number of individuals of each fish species collected per hour of electrofishing (CPUEN) at Reach 26 of the Mississippi River (Bnckhouse Slough) and at six reaches of the Illinois River Watenway in 1995.

\begin{tabular}{|c|c|c|c|c|c|c|c|c|}
\hline \multirow[b]{4}{*}{ Species } & \multicolumn{7}{|c|}{ Reach and Hours Fished } & \multirow{4}{*}{$\begin{array}{r}\text { Overall } \\
\text { CPUE } \\
2500\end{array}$} \\
\hline & \multirow{3}{*}{$\begin{array}{r}\text { Reach } 26 \\
100\end{array}$} & \multirow{3}{*}{$\begin{array}{l}\text { Alton } \\
500\end{array}$} & \multicolumn{4}{|c|}{ Starved } & \multirow{3}{*}{$\begin{array}{c}\text { Dresden } \\
200 \\
\end{array}$} & \\
\hline & & & La Grange & Peoria & Rock & Marseilles & & \\
\hline & & & 550 & 700 & 200 & 250 & & \\
\hline \multicolumn{9}{|l|}{ Lepisosteidae } \\
\hline shortnose gar & & & & 014 & & & & 004 \\
\hline \multicolumn{9}{|l|}{ Amıdae } \\
\hline bowfin & & 0.20 & & & & & & 004 \\
\hline Clupeidae & & & & & & & & \\
\hline gizzard shad & 5400 & 4260 & 88.73 & 12586 & 24250 & 9000 & 5050 & 9788 \\
\hline skıpjack hemng & & 020 & & 043 & & & & 016 \\
\hline Hiodontidae & & & & & & & & \\
\hline goldeye & 1.00 & 160 & 091 & & & & & 056 \\
\hline Cyprinidae & & & & & & & & \\
\hline bluntnose minnow & & 020 & 018 & & 23.00 & 2440 & 15000 & 1636 \\
\hline bulihead minnow & & 040 & 073 & 0.29 & 5900 & 5000 & 18650 & 2496 \\
\hline central stoneroller & & & & & & & 300 & 024 \\
\hline common carp & 400 & 340 & 2582 & 686 & 1.00 & 760 & 600 & 976 \\
\hline common carp $\times$ goldfish & & & & & & & 100 & 0.08 \\
\hline emerald shiner & 300 & 660 & 1109 & 1229 & 43850 & 7120 & 1050 & 5036 \\
\hline golden shiner & & & 036 & 557 & & 080 & 350 & 200 \\
\hline goldfish & & & 018 & 243 & & & & 0.72 \\
\hline grass carp & & & 018 & & & & & 004 \\
\hline minnow (unid) & & & 018 & & 350 & 360 & & 068 \\
\hline red shıner & 200 & 040 & 055 & 1.00 & 20.50 & 4240 & 7.00 & 7.00 \\
\hline sand shiner & & & & & 4300 & & & 344 \\
\hline silver chub & & & & 071 & & & & 0.20 \\
\hline spottail shiner & & & & 529 & 200 & 240 & & 188 \\
\hline suckermouth minnow & & & & & & 080 & & 008 \\
\hline Catostomıdae & & & & & & & & \\
\hline bigmouth buffalo & & 680 & 436 & 314 & & & & 320 \\
\hline black buffalo & & & 036 & & & & & 008 \\
\hline golden redhorse & & & & & 100 & 040 & 050 & 016 \\
\hline nver carpsucker & 900 & & & 586 & 150 & 0.80 & & 2.20 \\
\hline shorthead redhorse & & 020 & 145 & 0.71 & & 080 & & 064 \\
\hline smalimouth buffalo & 1800 & 500 & 655 & 1343 & 850 & 1.20 & 050 & 7.76 \\
\hline quillback & & & & 029 & 050 & & . & 0.12 \\
\hline Ictalundae & & & & & & & $\cdot$ & \\
\hline black bullhead & & & & 014 & & & 0.50 & 0.08 \\
\hline channel catfish & & 1020 & 309 & 200 & & 040 & 050 & 3.36 \\
\hline flathead catfish & & 140 & 091 & 014 & & & 050 & 0.56 \\
\hline yellow bullhead & & & & 029 & & 040 & & 0.12 \\
\hline Cypnnodontıdae & & & & & & & & \\
\hline blackstripe topminow & & & 018 & & & & 1.00 & 0.12 \\
\hline Poeciludae & & & & & & & & \\
\hline mosquitofish & & & 036 & & & & & 0.08 \\
\hline Athennidae & & & & & & & & \\
\hline brook silversıde & 100 & 020 & 018 & & & & & 0.12 \\
\hline Percichthyıdae & & & & & & & & \\
\hline stnped $\mathrm{x}$ white bass & & 020 & & 029 & & & & 0.12 \\
\hline white bass & 100 & 300 & 2091 & 829 & 150 & 040 & & 772 \\
\hline Centrarchıdae & & & & & & & & \\
\hline black crappie & 800 & 520 & 964 & 529 & 050 & & 050 & 5.04 \\
\hline bluegill & 4200 & 4480 & 4018 & 3986 & 15.00 & 4240 & 8300 & 4272 \\
\hline bluegill $x$ green sunfish & & & 0.18 & 100 & & & 050 & 036 \\
\hline green sunfish & 1.00 & 060 & 0.55 & 9.29 & 400 & 280 & 4600 & 716 \\
\hline largemouth bass & 500 & 7.60 & 473 & 1071 & & 960 & 1150 & 764 \\
\hline orangespotted suritish & 3.00 & 020 & & 100 & & 080 & 21.00 & 220 \\
\hline pumpkinseed & & 0.20 & & 0.14 & & & 050 & 012 \\
\hline redear sunfish & & & & & & 080 & & 008 \\
\hline rock bass & & & & & & & 650 & 0.52 \\
\hline smalimouth bass & & & & 0.57 & 1.00 & 120 & 900 & 108 \\
\hline varmouth & 1.00 & 0.20 & 109 & & & & & 032 \\
\hline white crappie & & 060 & 2.18 & 1.86 & 050 & 040 & & 1.20 \\
\hline Percidae & & & & & & & & \\
\hline logperch & & & & & 050 & & & 0.04 \\
\hline sauger & 1.00 & & 018 & 0.14 & & & & 012 \\
\hline walleye & & & & 0.14 & & & & 004 \\
\hline Sciaenidae & & & & & & & & \\
\hline freshwater drum & 3600 & 820 & 1509 & 25.57 & & 120 & & 1368 \\
\hline Total number per hour & 190.00 & 150.20 & 241.09 & 291.00 & 867.50 & 35680 & 60000 & 325.24 \\
\hline Number of species/hybrids & $17 / 0$ & $25 / 1$ & $28 / 1$ & $32 / 2$ & $19 / 0$ & $24 / 0$ & $23 / 1$ & $48 / 3$ \\
\hline
\end{tabular}


Table 9. Species ranked by relative abundance in number of fish collected per hour for 1995 . Species were added to the list in descending order of abundance until $95 \%$ of the total catch for that reach was obtained. Percentages are in parentheses.

\begin{tabular}{|c|c|c|c|c|c|c|}
\hline \multirow[b]{2}{*}{ Species } & \multicolumn{6}{|c|}{ Rankings by Reach } \\
\hline & Alton & La Grange & Peoria & $\begin{array}{l}\text { Starved } \\
\text { Rock }\end{array}$ & Marseilles & Dresden \\
\hline \multicolumn{7}{|l|}{ Clupeidae } \\
\hline gizzard shad & $2(28.4)$ & $1(36.8)$ & $1(43.3)$ & $2(28.0)$ & $1(25.2)$ & $4(8.4)$ \\
\hline \multicolumn{7}{|l|}{ Cyprinidae } \\
\hline bluntnose minnow & & & & $5(2.7)$ & $5(6.8)$ & $2(25.0)$ \\
\hline bullhead minnow & & & & $3(6.8)$ & $3(14.0)$ & $1(31.1)$ \\
\hline common carp & $10(2.3)$ & $3(10.7$ & $9(2.4)$ & & $7(2.1)$ & \\
\hline emerald shiner & $7(4.4)$ & $6(4.6)$ & $5(4.2)$ & $1(50.5)$ & $2(20.0)$ & $8(1.8)$ \\
\hline golden shiner & & & $11(1.9)$ & & & \\
\hline red shiner & & & & $6(2.4)$ & $4(11.9)$ & $10(1.2)$ \\
\hline sand shiner & & & & $4(5.0)$ & & \\
\hline spottail shiner & & & $12(1.8)$ & & & \\
\hline \multicolumn{7}{|l|}{ Catostomidae } \\
\hline bigmouth buffalo & $6(4.5)$ & $10(1.8)$ & $13(1.1)$ & & & \\
\hline river carpsucker & & & $10(2.0)$ & & & \\
\hline smallmouth buffalo & $9(3.3)$ & $8(2.7)$ & $4(4.6)$ & & & \\
\hline Ictaluridae & & & & & & \\
\hline channel catfish & $3(6.8)$ & $11(1.3)$ & & & & \\
\hline \multicolumn{7}{|l|}{ Percichthyidae } \\
\hline white bass & $11(2.0)$ & $4(8.7)$ & $8(2.8)$ & & & \\
\hline \multicolumn{7}{|l|}{ Centrarchidae } \\
\hline black crappie & $8(3.5)$ & $7(4.0)$ & $12(1.8)$ & & & \\
\hline bluegill & $1(29.8)$ & $2(16.7)$ & $2(13.7)$ & & $4(11.9)$ & $3(13.8)$ \\
\hline green sunfish & & & $7(3.2)$ & & & $5(7.7)$ \\
\hline largemouth bass & $5(5.1)$ & $9(2.0)$ & $6(3.7)$ & & $6(2.7)$ & $7(1.9)$ \\
\hline orangespotted sunfish & & & & & & $6(3.5)$ \\
\hline smallmouth bass & & & & & & $9(1.5)$ \\
\hline \multicolumn{7}{|l|}{ Sciaenidae } \\
\hline freshwater drum & $4(5.5)$ & $5(6.3)$ & $3(8.8)$ & & & \\
\hline \multicolumn{7}{|l|}{ Numbers of fishes } \\
\hline accounting for $95 \%$ & 11 & 11 & 14 & 6 & 8 & 10 \\
\hline
\end{tabular}


Table 10 Number of individuals of each fish species coltected per hour of electrofishing (CPUE ${ }_{N}$ ) at Reach 26 of the Mississippi

River (Bnckhouse Slough) and at six reaches of the Illinois River Waterway in 1996

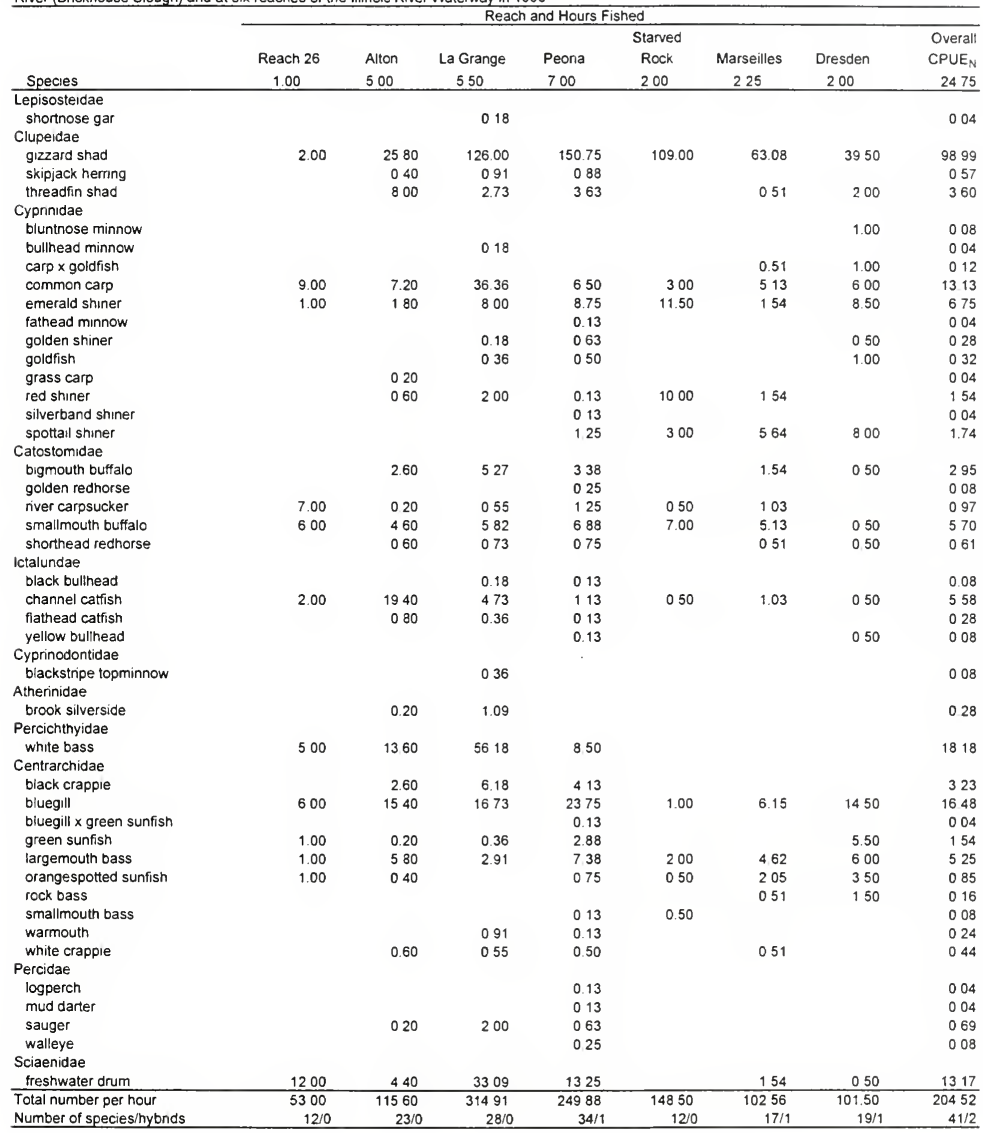


Table 11. Species ranked by relative abundance in number of fish collected per hour for 1996 . Species were added to the list in descending order of abundance until $95 \%$ of the total catch for that reach was obtained. Percentages are in parentheses.

\begin{tabular}{|c|c|c|c|c|c|c|}
\hline \multirow[b]{2}{*}{ Species } & \multicolumn{6}{|c|}{ Rankings by Reach } \\
\hline & Alton & La Grange & Peoria & $\begin{array}{l}\text { Starved } \\
\text { Rock }\end{array}$ & Marseilles & Dresden \\
\hline \multicolumn{7}{|l|}{ Clupeidae } \\
\hline gizzard shad & $1(22.3)$ & $1(40.0)$ & $1(60.3)$ & $1(73.4)$ & $1(61.5)$ & $1(38.9)$ \\
\hline threadfin shad & $5(6.9)$ & & $10(1.5)$ & & & $8(2.0)$ \\
\hline \multicolumn{7}{|l|}{ Cyprinidae } \\
\hline common carp & $6(6.2)$ & $3(11.5)$ & $8(2.6)$ & $5(2.0)$ & $4(5.0)$ & $5(5.9)$ \\
\hline emerald shiner & & $6(2.5)$ & $4(3.5)$ & $2(7.7)$ & $7(1.5)$ & $3(8.4)$ \\
\hline red shiner & & & & $3(6.7)$ & $7(1.5)$ & \\
\hline spottail shiner & & & & $5(2.0)$ & $3(5.5)$ & $4(7.9)$ \\
\hline \multicolumn{7}{|l|}{ Catostomidae } \\
\hline bigmouth buffalo & $10(2.2)$ & $8(1.7)$ & $11(1.4)$ & & $7(1.5)$ & \\
\hline smallmouth buffalo & $8(4.0)$ & $7(1.8)$ & $7(2.8)$ & $4(4.7)$ & $4(5.0)$ & \\
\hline \multicolumn{7}{|l|}{ Ictaluridae } \\
\hline channel catfish & $2(16.8)$ & $9(1.5)$ & & & & \\
\hline \multicolumn{7}{|l|}{ Percichthyidae } \\
\hline white bass & $4(11.8)$ & $2(17.8)$ & $5(3.4)$ & & & \\
\hline \multicolumn{7}{|l|}{ Centrarchidae } \\
\hline black crappie & $10(2.2)$ & $8(2.0)$ & $9(1.7)$ & & & \\
\hline bluegill & $3(13.3)$ & $5(5.3)$ & $2(9.5)$ & & $2(6.0)$ & $2(14.3)$ \\
\hline green sunfish & & & & & & $6(5.4)$ \\
\hline largemouth bass & $7(5.0)$ & & $6(3.0)$ & & $5(4.5)$ & $5(5.9)$ \\
\hline orangespotted sunfish & & & & & $6(2.0)$ & $7(3.4)$ \\
\hline rock bass & & & & & & $9(1.5)$ \\
\hline \multicolumn{7}{|l|}{ Sciaenidae } \\
\hline freshwater drum & $9(3.8)$ & $4(10.5)$ & $3(5.3)$ & & $7(1.5)$ & \\
\hline \multicolumn{7}{|l|}{ Number of fishes } \\
\hline accounting for $95 \%$ & 11 & 10 & 11 & 6 & 11 & 10 \\
\hline
\end{tabular}


Table 12 Numbers of individuals of each fish species collected per hour of electrofishing (CPUE $E_{N}$ ) on Reach 26 of the Mississippi

River (Bnckhouse Slough) and on six reaches of the Illinois River Waterway in 1997

\begin{tabular}{|c|c|c|c|c|c|c|c|c|}
\hline \multirow[b]{4}{*}{ Species } & \multicolumn{7}{|c|}{ Reach and Hours Fished } & \multirow{4}{*}{$\begin{array}{l}\text { Overall } \\
\text { CPUE }_{N} \\
2500 \\
\end{array}$} \\
\hline & \multirow{3}{*}{$\begin{array}{c}\text { Reach } 26 \\
100\end{array}$} & \multirow{3}{*}{$\begin{array}{l}\text { Alton } \\
500\end{array}$} & \multirow{3}{*}{$\begin{array}{c}\text { La Grange } \\
550\end{array}$} & \multicolumn{2}{|c|}{ Starved } & \multirow{3}{*}{$\begin{array}{c}\text { Marseilles } \\
225\end{array}$} & \multirow{3}{*}{$\begin{array}{c}\text { Dresden } \\
200\end{array}$} & \\
\hline & & & & Peona & Rock & & & \\
\hline & & & & 725 & 200 & & & \\
\hline \multicolumn{9}{|l|}{ Amudae } \\
\hline bowfin & & 020 & & & & & & 004 \\
\hline \multicolumn{9}{|l|}{ Cluperdae } \\
\hline gizzard shad & 1100 & 27.20 & 4636 & 9738 & 4900 & 4089 & 1400 & 5304 \\
\hline skipjack herrıng & & 060 & 182 & 028 & & 044 & & 064 \\
\hline threadfin shad & & 020 & & & & 622 & & 0.60 \\
\hline \multicolumn{9}{|l|}{ Cypnnidae } \\
\hline bluntnose minnow & & & & & 41.00 & 933 & 11500 & 1332 \\
\hline bullhead minnow & & & & 317 & 050 & 844 & & 172 \\
\hline central stoneroller & & & & & 050 & 044 & 300 & 032 \\
\hline common carp & 2000 & 1540 & 2309 & 16.14 & & 489 & 650 & 1460 \\
\hline common carp $x$ goldfish & & & & 041 & & & & 012 \\
\hline emerald shiner & & 3120 & 1255 & 2524 & 6950 & 57.78 & 10.00 & 2788 \\
\hline golden shiner & & & 036 & 041 & & & 150 & 032 \\
\hline goldfish & & & & 055 & & & 050 & 020 \\
\hline grass carp & & & & 041 & & & & 012 \\
\hline red shiner & 100 & 220 & & 138 & 2900 & 5200 & & 7.88 \\
\hline silver chub & 100 & & 0.18 & 014 & & & & 012 \\
\hline spottail shiner & & & & 938 & 2.00 & 044 & & 060 \\
\hline \multicolumn{9}{|l|}{ Catostomidae } \\
\hline bigmouth buffalo & 100 & 920 & 564 & 12.55 & & & & 676 \\
\hline golden redhorse & & & 018 & 014 & & 044 & 0.50 & 016 \\
\hline nver carpsucker & & 020 & 018 & 124 & & 044 & & 048 \\
\hline shorthead redhorse & & 080 & 109 & 124 & 150 & & 0.50 & 0.92 \\
\hline smallmouth buffalo & 1400 & 540 & 473 & 1434 & 650 & 222 & 150 & 7.68 \\
\hline \multicolumn{9}{|l|}{$\begin{array}{l}\text { Smalimoutn Durralo } \\
\text { Ictalundae }\end{array}$} \\
\hline channel catfish & 7.00 & 1320 & 582 & 207 & 050 & 222 & 050 & 508 \\
\hline flathead catfish & & 280 & 0.55 & 014 & & & 050 & 0.76 \\
\hline freckled madtom & & & 018 & & & & & 0.04 \\
\hline tadpole madtom & & & & & & 044 & & 004 \\
\hline \multicolumn{9}{|l|}{ Percichthyidae } \\
\hline stnped bass $x$ white bass & & & 018 & & & & & 004 \\
\hline white bass & 800 & 500 & 1455 & 1021 & & & & 7.48 \\
\hline \multicolumn{9}{|l|}{ Centrarchidae } \\
\hline black crappie & 200 & 300 & 455 & 690 & 050 & 044 & 1.50 & 388 \\
\hline bluegill & 2000 & 31.80 & 2236 & 2703 & 3.50 & 978 & 4800 & 2492 \\
\hline bluegill $\times$ green sunfish & & & & 014 & 0.50 & 044 & 10.50 & 096 \\
\hline green sunfish & & 220 & 018 & 3.72 & 200 & 489 & 1550 & 340 \\
\hline green $x$ orangespotted sunfish & & & & 0.14 & & & & 004 \\
\hline largemouth bass & 400 & 240 & 218 & 910 & 100 & 311 & 900 & 484 \\
\hline longear sunfish & & & & & & & 1.00 & 008 \\
\hline orangespotted sunfish & 2100 & 080 & 091 & 1.79 & & & 150 & 184 \\
\hline pumpkinseed & & & & 0.14 & & & & 0.04 \\
\hline rock bass & & & & & & & 2.00 & 0.16 \\
\hline smallmouth bass & & 040 & & 055 & & 089 & 100 & 040 \\
\hline white crappie & & 060 & 200 & 138 & & 044 & & 1.00 \\
\hline Percidae & & & & & & & & \\
\hline logperch & 1.00 & & & 124 & & & & 040 \\
\hline sauger & 2.00 & & 036 & 014 & & & & 020 \\
\hline Sciaenidae & & & & & & & & \\
\hline freshwater drum & 6500 & 1180 & 1527 & 2138 & & 178 & & 1468 \\
\hline Total number per hour & 17800 & 16660 & 165.27 & 26248 & 20750 & 20844 & 24400 & 20780 \\
\hline Number of species/hybrids & $15 / 0$ & $22 / 0$ & $23 / 1$ & $30 / 3$ & $14 / 1$ & $22 / 9$ & $20 / 1$ & $38 / 4$ \\
\hline
\end{tabular}


Table 13. Species ranked by relative abundance in number of fish collected per hour for 1997 . Species were added to the list in descending order of abundance until $95 \%$ of the total catch for that reach was obtained. Percentages are in parentheses.

\begin{tabular}{|c|c|c|c|c|c|c|}
\hline \multirow[b]{2}{*}{ Species } & \multicolumn{6}{|c|}{ Rankings by Reach } \\
\hline & Alton & La Grange & Peoria & $\begin{array}{l}\text { Starved } \\
\text { Rock }\end{array}$ & Marseilles & Dresden \\
\hline \multicolumn{7}{|l|}{ Clupeidae } \\
\hline $\begin{array}{l}\text { gizzard shad } \\
\text { threadfin shad }\end{array}$ & $3(16.3)$ & $1(28.1)$ & $1(37.1)$ & $2(23.6)$ & $\begin{array}{l}3(19.6) \\
7(3.0)\end{array}$ & $4(5.7)$ \\
\hline \multicolumn{7}{|l|}{ Cyprinidae } \\
\hline bluntnose minnow & & & & $3(19.8)$ & $5(4.5)$ & $1(47.1)$ \\
\hline $\begin{array}{l}\text { bullhead minnow } \\
\text { central stoneroller }\end{array}$ & & & $12(1.2)$ & & $6(4.1)$ & $9(1.2)$ \\
\hline common carp & $4(9.2)$ & $2(14.0)$ & $5(6.1)$ & & $8(2.3)$ & $8(2.7)$ \\
\hline emerald shiner & $2(18.7)$ & $6(7.6)$ & $3(9.6)$ & $1(33.5)$ & $1(27.1)$ & $6(4.1)$ \\
\hline red shiner & & & & $4(14.0)$ & $2(24.9)$ & \\
\hline \multicolumn{7}{|l|}{ Catostomidae } \\
\hline bigmouth buffalo & $7(5.5)$ & $8(3.4)$ & $7(4.8)$ & & & \\
\hline smallmouth buffalo & $8(3.2)$ & $9(2.9)$ & $6(5.5)$ & $5(3.1)$ & & \\
\hline \multicolumn{7}{|l|}{ Ictaluridae } \\
\hline channel catfish & $5(7.9)$ & $7(3.5)$ & $13(0.8)$ & & & \\
\hline flathead catfish & $11(1.7)$ & & & & & \\
\hline \multicolumn{7}{|l|}{ Percichthyidae } \\
\hline white bass & $9(3.0)$ & $5(8.8)$ & $8(3.9)$ & & & \\
\hline \multicolumn{7}{|l|}{ Centrarchidae } \\
\hline black crappie & $10(1.8)$ & $10(2.8)$ & $10(2.6)$ & & & \\
\hline bluegill & $1(19.1)$ & $3(13.5)$ & $2(10.3)$ & $6(1.7)$ & $4(4.7)$ & $2(19.7)$ \\
\hline bluegill $\times$ green sunfish & & & & & & $5(4.3)$ \\
\hline green sunfish & & & $11(1.4)$ & & $8(2.3)$ & $3(6.4)$ \\
\hline largemouth bass & $12(1.4)$ & $11(1.3)$ & $9(3.5)$ & & $9(1.5)$ & $7(3.7)$ \\
\hline \multicolumn{7}{|l|}{ Sciaenidae } \\
\hline freshwater drum & $6(7.1)$ & $4(9.2)$ & $4(8.1)$ & & & \\
\hline \multicolumn{7}{|l|}{ Number of fishes } \\
\hline accounting for $95 \%$ & 12 & 11 & 13 & 6 & 10 & 9 \\
\hline
\end{tabular}


Table 14 Numbers of individuals of each fish species collected per hour of electrofishing (CPUEN) on Reach 26 of the Mississippi River (Bnckhouse Slough) and on six reaches of the llinois River Waterway in 1998

\begin{tabular}{|c|c|c|c|c|c|c|c|c|}
\hline \multirow[b]{4}{*}{ Species } & \multirow{2}{*}{\multicolumn{7}{|c|}{ Reach and Hours Fished }} & \multirow{3}{*}{$\begin{array}{l}\text { Overall } \\
\text { CPUE }\end{array}$} \\
\hline & & & & & & & & \\
\hline & \multirow{2}{*}{$\begin{array}{c}\text { Reach } 26 \\
100\end{array}$} & \multirow{2}{*}{$\begin{array}{l}\text { Aiton } \\
500 \\
\end{array}$} & \multirow{2}{*}{$\begin{array}{l}\text { La Grange } \\
550 \\
\end{array}$} & \multirow{2}{*}{$\begin{array}{l}\text { Peona } \\
800 \\
\end{array}$} & \multirow{2}{*}{$\begin{array}{l}\text { Rock } \\
200 \\
\end{array}$} & \multirow{2}{*}{$\begin{array}{c}\text { Marselles } \\
275 \\
\end{array}$} & \multirow{2}{*}{$\begin{array}{l}\text { Dresden } \\
175 \\
\end{array}$} & \\
\hline & & & & & & & & 2600 \\
\hline Cluperdae & & & & & & & & \\
\hline gizzard shad & 3300 & 2360 & 5018 & 6538 & 5800 & 2036 & 2114 & 4458 \\
\hline skıpjack hemng & & & 036 & 038 & 050 & & & 023 \\
\hline threadfin shad & & 200 & 073 & 313 & & & & 150 \\
\hline Hrodontidae & & & & & & & & \\
\hline goideye & & & 018 & & & & & 004 \\
\hline Cyprinıdae & & & & & & & & \\
\hline bullhead minnow & & 020 & & 125 & 750 & 182 & 057 & 123 \\
\hline bluntnose minnow & 100 & & 018 & 063 & 250 & 509 & 1429 & 196 \\
\hline common carp & 400 & 880 & 2436 & 913 & 150 & 400 & 400 & 1062 \\
\hline common carp $\times$ goldfish & & & & 025 & & & & 008 \\
\hline emeraid shiner & 700 & 1520 & 091 & 150 & 10950 & 4291 & 571 & 1719 \\
\hline golden shiner & 100 & & & & & & 057 & 008 \\
\hline goldfish & & & 036 & 038 & & & & 019 \\
\hline grass carp & & 020 & & 013 & & & & 008 \\
\hline red shiner & & 020 & 018 & & & & & 008 \\
\hline sand shiner & & & & 013 & & & & 004 \\
\hline silver chub & & & & 013 & & & & 004 \\
\hline silverband shıner & & 020 & 055 & & & & & 015 \\
\hline spotfin shiner & & 020 & & & 700 & 1236 & & 188 \\
\hline spottall shiner & & & & 225 & 850 & 036 & & 138 \\
\hline Catostomidae & & & & & & & & \\
\hline bigmouth buffalo & & 360 & 327 & 613 & & & & 327 \\
\hline black buffalo & & & 018 & 025 & & & & 012 \\
\hline golden redhorse & & & & 038 & 100 & 109 & 114 & 0.38 \\
\hline quillback & & & 036 & & 300 & & 057 & 035 \\
\hline river carpsucker & 300 & 020 & 036 & 213 & & 036 & & 092 \\
\hline shorthead redhorse & & & 091 & 075 & & & & 042 \\
\hline smallmouth buffalo & & 100 & 618 & 1138 & 1700 & 545 & 457 & 719 \\
\hline white sucker & & & 018 & & & & & 004 \\
\hline Ictalundae & & & & & & & & \\
\hline channel cattish & 500 & 820 & 455 & 238 & 100 & 036 & 286 & 377 \\
\hline flathead catfish & & 220 & 182 & 113 & & & & $\uparrow 15$ \\
\hline Cypnnodontıdae & & & & & & & & \\
\hline blackstnpe topminnow & & 020 & & & & & 286 & 023 \\
\hline Poecilıdae & & & & & & & & \\
\hline mosquitofish & & & & 013 & & & & 004 \\
\hline Percichthyidae & & & & & & & & \\
\hline white bass & 600 & 600 & 2109 & 1088 & 200 & 145 & & 950 \\
\hline yellow bass & & & 018 & & & & & 004 \\
\hline Centrarchidae & & & & & & & & \\
\hline black crappie & & 040 & 200 & 675 & 150 & 109 & & 281 \\
\hline bluegill & 1500 & 1040 & 1273 & 2675 & 200 & 109 & 1429 & 1473 \\
\hline bluegill $\times$ green sunfish & & 020 & & 163 & & & 571 & 092 \\
\hline bluegill $x$ orangespotted sunfish & & & & & & 109 & 057 & 015 \\
\hline green sunfish & & 040 & 055 & 1225 & 250 & 255 & 3143 & 654 \\
\hline largemouth bass & 1.00 & 1.40 & 327 & 875 & 250 & 327 & 286 & 442 \\
\hline orangespotted sunfish & 1900 & 020 & & 313 & & & 114 & 181 \\
\hline pumpkinseed & & & & & & 036 & & 004 \\
\hline smallmouth bass & & & & 038 & 050 & & 171 & 027 \\
\hline warmouth & & & 018 & 025 & & & & 012 \\
\hline white crappie & & & 018 & 088 & & & & 0.31 \\
\hline Percidae & & & & & & & & \\
\hline mud darter & & 020 & & & & & & 004 \\
\hline sauger & 100 & 020 & 200 & 100 & & & & 081 \\
\hline slenderhead darter & & & & 013 & & & & 004 \\
\hline Scıaenıdae & & & & & & & & \\
\hline freshwater drum & 1500 & 720 & 2291 & 1663 & 050 & 073 & 114 & 1212 \\
\hline Total number per hour & 11100 & 9260 & 16091 & 19863 & 22850 & 10582 & 11714 & 15388 \\
\hline Number of species/hybrids & $13 / 0$ & $24 / 1$ & $29 / 0$ & $33 / 2$ & $19 / 3$ & $18 / 1$ & $17 / 2$ & $44 / 3$ \\
\hline
\end{tabular}


Table 15. Species ranked by relative abundance in number of fish collected per hour for 1998 . Species were added to the list in descending order of abundance until $95 \%$ of the total catch for that reach was obtained. Percentages are in parentheses.

\begin{tabular}{|c|c|c|c|c|c|c|}
\hline \multirow[b]{2}{*}{ Species } & \multicolumn{6}{|c|}{ Rankings by Reach } \\
\hline & Alton & La Grange & Peoria & $\begin{array}{l}\text { Starved } \\
\text { Rock }\end{array}$ & Marseilles & Dresden \\
\hline \multicolumn{7}{|l|}{ Clupeidae } \\
\hline gizzard shad & $1(25.1)$ & $1(31.2)$ & $1(32.9)$ & $2(25.4)$ & $2(19.2)$ & $2(18.0)$ \\
\hline threadfin shad & $10(2.2)$ & & $11(1.6)$ & & & \\
\hline \multicolumn{7}{|l|}{ Cyprinidae } \\
\hline bullhead minnow & & & & $5(3.3)$ & $9(1.7)$ & \\
\hline bluntnose minnow & & & & $8(1.1)$ & $5(4.8)$ & $3(12.2)$ \\
\hline common carp & $4(9.5)$ & $2(15.1)$ & $7(4.6)$ & & $6(3.8)$ & $8(3.4)$ \\
\hline emerald shiner & $2(16.4)$ & & & $1(47.9)$ & $1(40.5)$ & $5(4.9)$ \\
\hline spotfin shiner & & & & $6(3.1)$ & $3(11.7)$ & \\
\hline spottail shiner & & & $14(1.1)$ & $4(3.7)$ & & \\
\hline \multicolumn{7}{|l|}{ Catostomidae } \\
\hline bigmouth buffalo & $8(3.9)$ & $8(2.0)$ & $10(3.1)$ & & & \\
\hline quillback & & & & $7(1.3)$ & & \\
\hline river carpsucker & & & $15(1.1)$ & & & \\
\hline smallmouth buffalo & & $6(3.8)$ & $5(5.7)$ & $3(7.4)$ & $4(5.2)$ & $7(3.9)$ \\
\hline \multicolumn{7}{|l|}{ Ictaluridae } \\
\hline channel catfish & $5(8.9)$ & $7(2.8)$ & $13(1.2)$ & & & $9(2.4)$ \\
\hline flathead catfish & $9(2.4)$ & $12(1.1)$ & & & & \\
\hline \multicolumn{7}{|l|}{ Cyprinodontidae } \\
\hline blackstripe topminnow & & & & & & $9(2.4)$ \\
\hline \multicolumn{7}{|l|}{ Percichthyidae } \\
\hline white bass & $7(6.5)$ & $4(13.1)$ & $6(5.5)$ & & $10(1.4)$ & \\
\hline \multicolumn{7}{|l|}{ Centrarchidae } \\
\hline black crappie & & $10(1.2)$ & $9(3.4)$ & & & \\
\hline bluegill & $3(11.2)$ & $5(7.9)$ & $2(13.5)$ & & & $3(12.2)$ \\
\hline bluegill $x$ green sunfish & & & $16(0.8)$ & & & $5(4.9)$ \\
\hline green sunfish & & & $4(6.2)$ & $8(1.1)$ & $8(2.4)$ & $1(26.8)$ \\
\hline $\begin{array}{l}\text { largemouth bass } \\
\text { orangespotted sunfish }\end{array}$ & $11(1.5)$ & $8(2.0)$ & $\begin{array}{l}8(4.4) \\
11(1.6)\end{array}$ & $8(1.1)$ & $7(3.1)$ & $9(2.4)$ \\
\hline smallmouth bass & & & & & & $12(1.5)$ \\
\hline \multicolumn{7}{|l|}{ Percidae } \\
\hline sauger & & $10(1.2)$ & & & & \\
\hline \multicolumn{7}{|l|}{ Sciaenidae } \\
\hline freshwater drum & $6(7.8)$ & $3(14.2)$ & $3(8.4)$ & & & \\
\hline \multicolumn{7}{|l|}{ Number of fishes } \\
\hline accounting for $95 \%$ & 11 & 12 & 16 & 10 & 10 & 12 \\
\hline
\end{tabular}


Mississippi River. Of these fishes, 25, 278 individuals were collected from the Illinois Waterway sites, and 643 were collected from Brickhouse Slough of the Mississippi River. These results are similar to the first five years of F-101-R (1989-1993), when 60 species of fishes were collected representing 12 families (Lerczak et al. 1995:

25). The year with the greatest overall catch of fishes was 1995 (7941 individuals, CPUE $_{N}$ 325) (Table 8) and the year with the fewest overall catch of fishes was 1994 (3421 individuals, CPUE $_{\mathrm{N}}$ 131) (Table 6).

For all stations combined, the greatest number of species were collected in 1995 (48 species plus 3 hybrids) and the least were in 1997 (38 species plus 4 hybrids) (Tables 8 and 12, respectively). The number of species collected from upper waterway reaches ranged from 12 for Starved Rock in 1996 (Table 10) to 24 for Marseilles in 1995 (Table 8). The number of species collected from middle river reaches ranged from 23 for La Grange Reach in 1997 (Table 12) to 34 for Peoria Reach in 1996 (Table 10). The number of species collected from the lower river (Alton Reach) ranged from 18 in 1994 (Table 6) to 25 (Table 8) in 1995. The Peoria Reach consistantly had highest species richness during all years (1994-1998) of sampling.

Rankings by Relative Abundance. Rankings by relative abundance in number of fish collected per hour highlight the consistent dominance by gizzard shad during all years except 1994, when small cyprinids (emerald shiner and bluntnose minnow) and bluegill were most numerous (Tables 7, 9, 11, 13, and 15). Gizzard 
shad ranked first in numerical abundance in La Grange, Peoria, and Marseilles reaches in 1995, all reaches in 1996, La Grange and Peoria reaches in 1997, and Alton, La Grange and Peoria reaches in 1998 (Tables 9, 11, 13, and 15). Other numerically important species have included bluntnose minnow, bullhead minnow, emerald shiner, bluegill, and green sunfish. Bluntnose minnow ranked first in numerical abundance in Dresden Reach in 1994 and 1997 (Tables 7 and 13). Bullhead minnow ranked first in numerical abundance in Dresden Reach in 1995 (Table 9). Emerald shiner ranked first in numerical abundance in Starved Rock and Marseilles reaches during all years of this project except 1996 (Tables 7, 9, 13, and 15). Bluegill ranked first in numerical abundance in Alton, La Grange, and Peoria reaches in 1994 and in Alton Reach in 1995 and 1997 (Tables 7, 9, and 13). Green sunfish ranked first in numerical abundance in Dresden Reach in 1998 (Table 15).

\section{CPUE $_{N}$ of Five Most Numerically Abundant Species. Catch rates in} numbers of individuals collected per hour by electrofishing for the top five most numerically abundant species are shown in Figures 2 through 6 for the lower, middle, and upper Illinois waterway reaches. For gizzard shad, a similar trend was noticed in all three river sections (Figure 2). Lowest gizzard shad CPUE $_{N}$ occurred in 1994 in all sections, and was highest in 1995 (lower and upper river) and 1996 (middle river), prior to a decline in $\mathrm{CPUE}_{\mathrm{N}}$ in 1996, 1997, and 1998. Overall, catches of gizzard shad have been much higher in the middle and upper river than in the lower river. Catches of common carp have been highest $\left(\mathrm{CPUE}_{\mathrm{N}} 15-20\right)$ in the middle Illinois 


\section{gizzard shad}

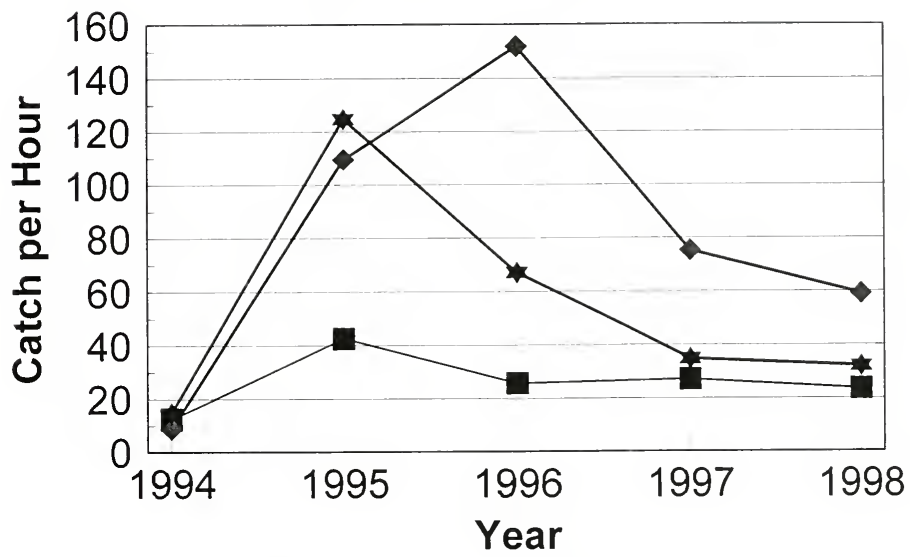

lower river middle river upper river
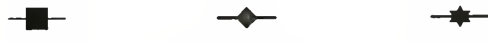

Figure 2. Catch per hour of gizzard shad from 1994 through 1998 in the lower (Alton Reach), middle (La Grange and Peoria reaches), and upper (Starved Rock, Marseilles, and Dresden reaches) Illinois River waterway. 


\section{common carp}

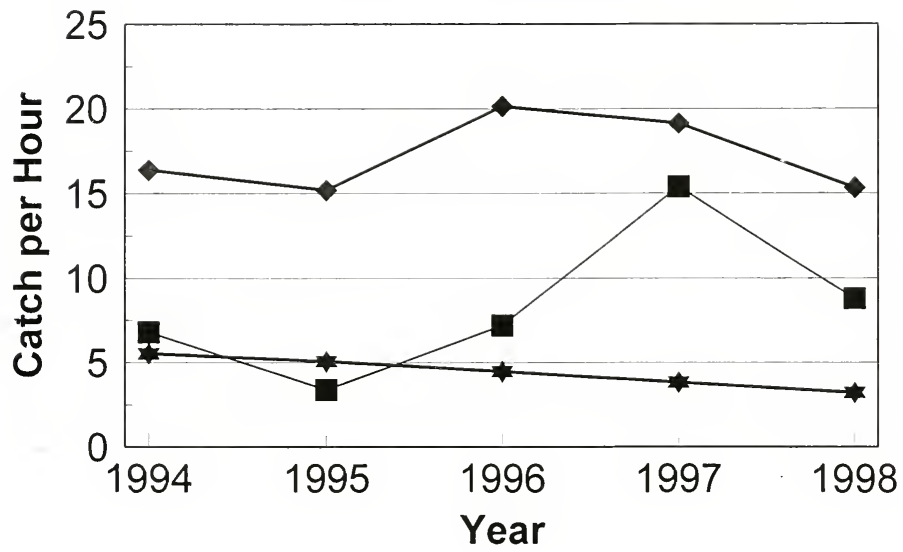

lower river middle river upper river
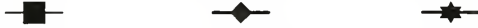

Figure 3. Catch per hour of common carp from 1994 through 1998 in the lower (Alton Reach), middle (La Grange and Peoria reaches), and upper (Starved Rock, Marseilles, and Dresden reaches) Illinois River waterway. 


\section{emerald shiner}

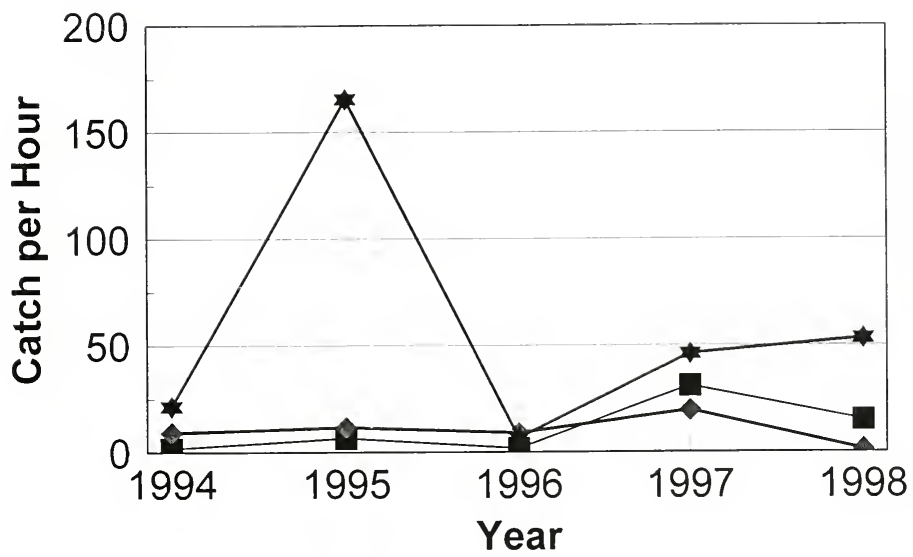

lower river middle river upper river
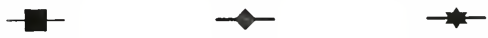

Figure 4. Catch per hour of emerald shiner from 1994 through 1998 in the lower (Alton Reach), middle (La Grange and Peoria reaches), and upper (Starved Rock, Marseilles, and Dresden reaches) Illinois River waterway. 


\section{bluegill}

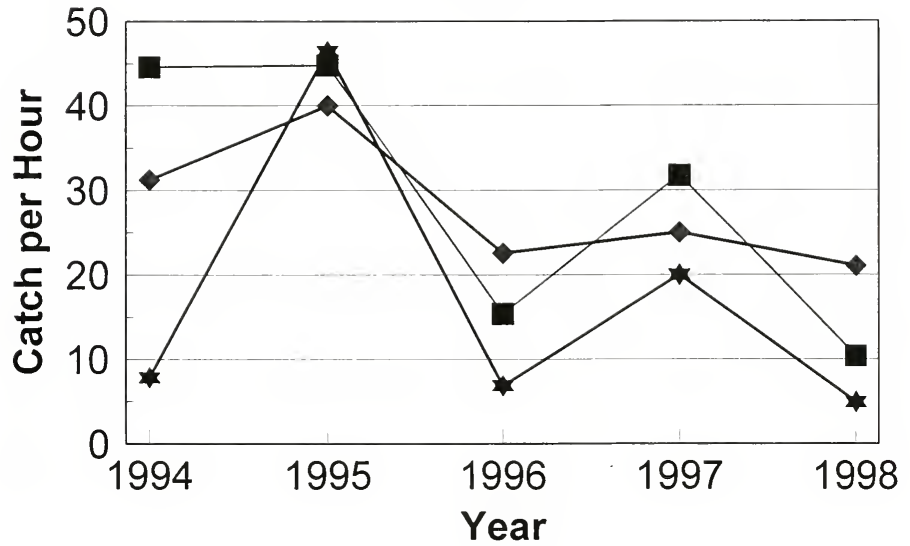

lower river middle river upper river
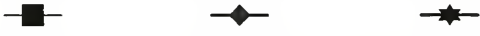

Figure 5. Catch per hour of bluegill from 1994 through 1998 in the lower (Alton Reach), middle (La Grange and Peoria reaches), and upper (Starved Rock, Marseilles, and Dresden reaches) Illinois River waterway. 


\section{freshwater drum}

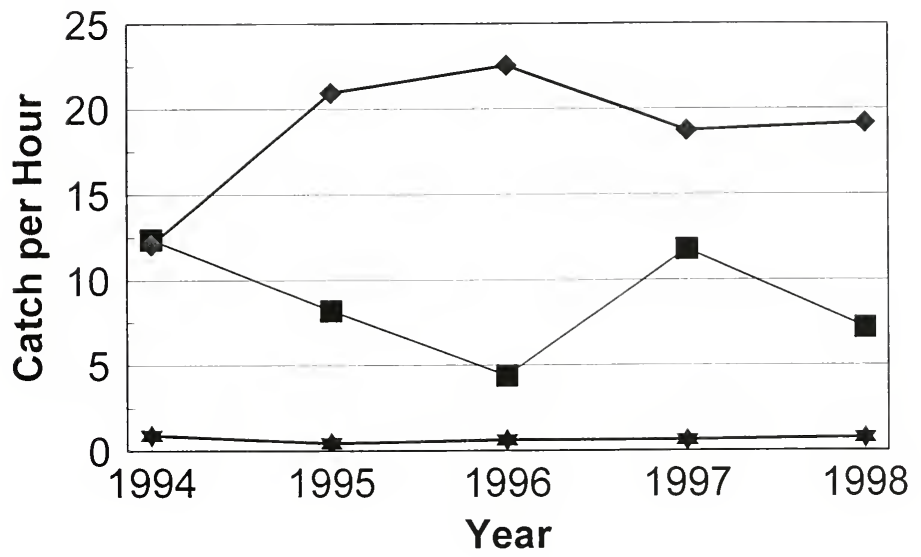

lower river middle river upper river $\rightarrow$
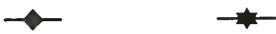

Figure 6. Catch per hour of freshwater drum from 1994 through 1998 in the lower (Alton Reach), middle (La Grange and Peoria reaches), and upper (Starved Rock, Marseilles, and Dresden reaches) Illinois River waterway. 
River reaches (Figure 3). Far fewer common carp have been taken in the upper river, where $\mathrm{CPUE}_{\mathrm{N}}$ has been consistantly low during the five years of this project; only 5 common carp per hour or less have been collected from 1994 through 1998. Catches of emerald shiner have typically been less than $\mathrm{CPUE}_{\mathrm{N}} 50$ except in the upper river reaches in 1995, when average CPUE $_{N}$ was 165 (Figure 4). Catches of bluegill in all three river sections have shown similar trends over the five years of this study (Figure 5). Bluegill CPUE $E_{N}$ was highest in $1995\left(\right.$ CPUE $\left._{N} 40-46\right)$ and has exhibited an annual cyclical pattern in population size. This pattern appears to be consistant throughout the entire waterway. Catches of freshwater drum have been highest in the middle river reaches $\left(\mathrm{CPUE}_{\mathrm{N}}\right.$ 12-23) (Figure 6). Collections in the lower river have ranged from $\mathrm{CPUE}_{\mathrm{N}} 5-12$, and in the upper river have been extremely low by comparison, with $\mathrm{CPUE}_{\mathrm{N}}<1$ during all years of this project.

\section{Catch Rates in Weights (pounds) Collected per Hour by Reach.}

Catch rates in pounds of fish collected per hour $\left(\mathrm{CPUE}_{\mathrm{W}}\right)$ were also examined to provide an estimation of fish biomass and production of each Illinois River reach. Overall, CPUE ${ }_{W}$ ranged from 48 pounds per hour in 1995 to 74 pounds per hour in 1997 (Tables 16-25). La Grange Reach has consistantly provided the highest catches in weight (CPUE $\left.{ }_{W} 81-108\right)$ except in 1997, when average hourly collections from Peoria Reach were 114 pounds (Table 22). Lowest catches in weight each year have typically come from Starved Rock $\left(C P U E_{W} 11-32\right)$ and Marseilles (CPUE $E_{W}$ 
Table 16. Pounds of each fish species collected per hour of electrofishing (CPUE ${ }_{W}$ ) at six reaches of the Illinois River Waterway in 1994. Pounds per hour less than 0.01 are indicated by 0.00

\begin{tabular}{|c|c|c|c|c|c|c|c|}
\hline \multirow[b]{2}{*}{ Species } & \multicolumn{6}{|c|}{ Reach and Hours Fished } & \multirow[b]{2}{*}{$\begin{array}{l}\text { Overall } \\
\text { CPUE } \\
2695 \\
\end{array}$} \\
\hline & $\begin{array}{r}\text { Alton } \\
5.00 \\
\end{array}$ & $\begin{array}{r}\text { La Grange } \\
8.50\end{array}$ & $\begin{array}{r}\text { Peoria } \\
6.95\end{array}$ & $\begin{array}{r}\text { Starved } \\
\text { Rock } \\
2.00\end{array}$ & $\begin{array}{r}\text { Marseilles } \\
2.50\end{array}$ & $\begin{array}{r}\text { Dresden } \\
2.00\end{array}$ & \\
\hline \multicolumn{8}{|l|}{ Lepisosteidae } \\
\hline shortnose gar & & 0.04 & & & & & 0.01 \\
\hline $\begin{array}{l}\text { Amiidae } \\
\text { bowfin }\end{array}$ & 0.44 & & & & & & 0.08 \\
\hline \multicolumn{8}{|l|}{ Clupeidae } \\
\hline gizzard shad & 0.99 & 1.49 & 0.74 & 1.85 & 1.75 & 1.98 & 1.29 \\
\hline shipjack herring & & & 0.24 & & 0.02 & & 006 \\
\hline threadfin shad & 0.01 & & 0.02 & & & & 0.01 \\
\hline \multicolumn{8}{|l|}{ Hiodontidae } \\
\hline goldeye & & 0.05 & 0.11 & & & & 0.04 \\
\hline \multicolumn{8}{|l|}{ Cyprinidae } \\
\hline bluntnose minnow & & & & 0.00 & 0.06 & 0.21 & 0.02 \\
\hline bullhead minnow & & 0.00 & 0.00 & 0.02 & 0.00 & 0.02 & 0.00 \\
\hline carp $\times$ goldfish & & & & & 0.86 & 4.38 & 040 \\
\hline common carp & 21.91 & 47.95 & 12.30 & 9.14 & 546 & 20.97 & 25.10 \\
\hline emerald shiner & 0.00 & 0.00 & 0.03 & 0.07 & 0.10 & 0.07 & 0.03 \\
\hline golden shiner & & & & & 0.00 & 0.05 & 0.00 \\
\hline goldfish & & 0.12 & 0.15 & & & & 0.08 \\
\hline minnow (unid.) & & & 0.00 & & 0.00 & 0.00 & 0.00 \\
\hline red shiner & & 0.00 & & 0.00 & 0.00 & & 0.00 \\
\hline sand shiner & & & & 0.01 & 0.01 & & 0.00 \\
\hline silverchub & & & 0.02 & & & & 0.01 \\
\hline spottail shıner & & & 0.00 & & 0.01 & 0.10 & 0.01 \\
\hline \multicolumn{8}{|l|}{ Catostomidae } \\
\hline bigmouth buffalo & 4.37 & 1850 & 12.36 & & & & 9.83 \\
\hline golden redhorse & & 0.00 & 0.35 & 0.22 & 0.61 & 0.52 & 020 \\
\hline highfin carpsucker & & & & 0.37 & & & 0.03 \\
\hline northem hog sucker & & & & & 0.02 & & 0.00 \\
\hline quillback & . & & 0.17 & & & & 0.04 \\
\hline river carpsucker & ${ }^{\circ}$ & 0.16 & 4.14 & 0.38 & 1.37 & 0.85 & 1.34 \\
\hline shorthead redhorse & & 0.64 & 1.27 & & & 0.03 & 0.53 \\
\hline smallmouth buffalo & 1.05 & 3.18 & 5.15 & 1211 & 3.26 & & 3.73 \\
\hline \multicolumn{8}{|l|}{ Ictaluridae } \\
\hline channel catfish & 18.59 & 7.11 & 248 & 451 & 1.27 & & 6.78 \\
\hline flathead catfish & 0.56 & 1.44 & 0.44 & & & 4.35 & 0.99 \\
\hline yellow bullihead & 0.17 & & & & & & 0.03 \\
\hline \multicolumn{8}{|l|}{ Atherinidae } \\
\hline brook silverside & & & & & & 0.00 & 0.00 \\
\hline Percichthyidae & & & & & & & \\
\hline white bass & 0.41 & 1.56 & 1.23 & 0.47 & 0.46 & & 0.96 \\
\hline Centrarchidae & & & & & & & \\
\hline black crappie & 2.40 & 3.67 & 0.30 & & & 0.38 & 1.71 \\
\hline bluegill & 2.70 & 1.90 & 2.20 & 0.03 & 0.54 & 0.77 & 1.78 \\
\hline bluegill $\times$ green sunfish & & & 0.19 & & & 0.00 & 0.05 \\
\hline green sunfish & 0.04 & 0.04 & 1.23 & 0.06 & 0.17 & 0.36 & 0.38 \\
\hline largemouth bass & 6.43 & 5.53 & 3.02 & 0.21 & 4.20 & 0.21 & 4.14 \\
\hline orangespotted sunfish & & 0.00 & 0.01 & 0.00 & & 0.01 & 0.00 \\
\hline pumpkinseed & & & & & & 0.06 & 0.00 \\
\hline rock bass & & & & & & 0.18 & 0.01 \\
\hline smallmouth bass & & 0.00 & 0.02 & 0.07 & 0.11 & 0.80 & 0.08 \\
\hline warmouth & 0.05 & 0.01 & & & & & 0.01 \\
\hline white crappie & & 0.33 & 0.12 & & & & 0.14 \\
\hline Percidae & & & & & & & \\
\hline sauger & 0.03 & 0.14 & 0.12 & & & & 0.08 \\
\hline slenderhead darter & & & & & 0.00 & & 0.00 \\
\hline Sciaenidae & & & & & & & \\
\hline freshwater drum & 2.28 & 1.29 & 2.15 & & 0.66 & 0.88 & 1.51 \\
\hline Total pounds per hour & 62.42 & 95.17 & 50.56 & 29.50 & 20.95 & 37.24 & 61.52 \\
\hline
\end{tabular}


Table 17. Species ranked by relative abundance in pounds of fish collected per hour for 1994 . Species were added to the list in descending order of abundance until $95 \%$ of the total catch for that reach was obtained. Percentages are in parentheses.

\begin{tabular}{|c|c|c|c|c|c|c|}
\hline \multirow[b]{2}{*}{ Species } & \multicolumn{6}{|c|}{ Rankings by Reach } \\
\hline & Alton & La Grange & Peoria & $\begin{array}{l}\text { Starved } \\
\text { Rock }\end{array}$ & Marseilles & Dresden \\
\hline \multicolumn{7}{|l|}{ Clupeidae } \\
\hline gizzard shad & & $9(1.6)$ & $11(1.4)$ & $4(6.3)$ & $4(8.4)$ & $4(5.3)$ \\
\hline \multicolumn{7}{|l|}{ Cyprinidae } \\
\hline carp $\times$ goldfish & & & & & $7(4.1)$ & $2(11.8)$ \\
\hline common carp & $1(35.1)$ & $1(50.4)$ & $2(24.3)$ & $2(31.0)$ & $1(26.1)$ & $1(56.3)$ \\
\hline \multicolumn{7}{|l|}{ Catostomidae } \\
\hline bigmouth buffalo & $4(7.0)$ & $2(19.4)$ & $1(24.4)$ & & & \\
\hline golden redhorse & & & & & $9(2.9)$ & $9(1.4)$ \\
\hline river carpsucker & & & $4(8.2)$ & & $4(6.5)$ & $6(2.3)$ \\
\hline shorthead redhorse & & & $9(2.5)$ & & & \\
\hline smallmouth buffalo & $8(1.7)$ & $6(3.3)$ & $3(10.2)$ & $1(41.1)$ & $3(15.6)$ & \\
\hline \multicolumn{7}{|l|}{ Ictaluridae } \\
\hline channel catfish & $2(29.8)$ & $3(7.5)$ & $6(4.9)$ & $3(15.3)$ & $6(6.1)$ & \\
\hline flathead catfish & & & & & & $3(11.7)$ \\
\hline \multicolumn{7}{|l|}{ Percichthyidae } \\
\hline white bass & & $8(1.7)$ & $10(2.4)$ & $5(1.6)$ & & \\
\hline \multicolumn{7}{|l|}{ Centrarchidae } \\
\hline black crappie & $6(3.8)$ & $5(3.9)$ & & & & \\
\hline $\begin{array}{l}\text { bluegill } \\
\text { green sunfish }\end{array}$ & $5(4.3)$ & $7(2.0)$ & $\begin{array}{l}7(4.4) \\
10(2.4)\end{array}$ & & $10(2.6)$ & $8(2.1)$ \\
\hline largemouth bass & $3(10.3)$ & $4(5.8)$ & $5(6.0)$ & & $2(20.0)$ & \\
\hline smallmouth bass & & & & & & $7(2.1)$ \\
\hline \multicolumn{7}{|l|}{ Sciaenidae } \\
\hline freshwater drum & $7(3.7)$ & & $8(4.3)$ & & $8(3.2)$ & $5(2.4)$ \\
\hline \multicolumn{7}{|l|}{ Numbers of fishes } \\
\hline accounting for $95 \%$ & 8 & 9 & 12 & 5 & 10 & 9 \\
\hline
\end{tabular}


Table 18. Pounds of each fish species collected per hour of electrofishng (CPUEW) at SIx reaches of the Illinois River Waterway in 1995 Pounds per hour less than 0.01 are indicated by 000

\begin{tabular}{|c|c|c|c|c|c|c|c|}
\hline - & & $\mathrm{F}$ & $\mathrm{h}$ and $\mathrm{HOU}$ & Fished & & & \\
\hline & & & & Starved & & & Overalt \\
\hline & Alton & La Grange & Peona & Rock & Marseilles & Dresden & CPUE \\
\hline Species & 500 & 550 & 7.00 & 200 & 250 & 200 & 2400 \\
\hline Lepisosteidae & & & & & & & \\
\hline shortnose gar & & & 0.13 & & & & 004 \\
\hline Amiidae & & & & & & & \\
\hline bowfin & 075 & & & & & & 016 \\
\hline Clupeıdae & & & & & & & \\
\hline gizzard shad & 1.05 & 146 & 282 & 530 & 337 & 307 & 242 \\
\hline skipjack hemng & 002 & & 0.02 & & & & 001 \\
\hline Hiodontidae & & & & & & & \\
\hline goldeye & 021 & 0.05 & & & & & 006 \\
\hline Cypnnidae & & & & & & & \\
\hline bluntnose minnow & 000 & 000 & & 0.05 & 0.10 & 036 & 004 \\
\hline bullhead minnow & 000 & 000 & 000 & 008 & 009 & 044 & 005 \\
\hline carp $\times$ goldfish & & & & & & 1.12 & 009 \\
\hline central stoneroller & & & & & & 002 & 000 \\
\hline common carp & 663 & 4784 & 734 & 1.79 & 747 & 1151 & 1637 \\
\hline emerald shıner & 001 & 002 & 003 & 139 & 016 & 0.02 & 015 \\
\hline golden shiner & & 000 & 0.03 & & 000 & 002 & 001 \\
\hline goldfish & & 001 & 013 & & & & 004 \\
\hline grass carp & & 124 & & & & & 028 \\
\hline minnow (unid.) & & 000 & & 0.00 & 000 & & 000 \\
\hline red shıner & 000 & 0.00 & 001 & 002 & 0.11 & 002 & 002 \\
\hline sand shiner & & & & 004 & & & 000 \\
\hline silverchub & & & 0.00 & & & & 000 \\
\hline spottail shiner & & & 001 & 000 & 002 & & 0.01 \\
\hline suckermouth mınnow & & & & & 001 & & 0.00 \\
\hline Catostomidae & & & & & & & \\
\hline bigmouth buffalo & 1827 & 1129 & 763 & & & & 862 \\
\hline black buffalo & & 0.61 & & & & & 014 \\
\hline golden redhorse & & & & 0.11 & 005 & 0.06 & 002 \\
\hline nver carpsucker & & & 1.99 & 0.62 & 060 & & 069 \\
\hline shorthead redhorse & 0.03 & 026 & 0.15 & & 0.50 & & 016 \\
\hline smailmouth buffalo & 219 & 289 & 437 & 842 & 147 & 0.55 & 3.29 \\
\hline quillback & & & 001 & 045 & & & 0.04 \\
\hline Ictalundae & & & & & & & \\
\hline black bullhead & & & 0.02 & & & 0.00 & 001 \\
\hline channel catfish & 890 & 188 & 1.60 & & 091 & $0 \infty 0$ & 285 \\
\hline flathead catfish & 053 & 147 & 002 & & & 617 & 097 \\
\hline yellow bullhead & & & 010 & & 0.12 & & 0.04 \\
\hline Cypnnodontudae & & & & & & & \\
\hline blackstnpe topminow & & 000 & & & & 000 & 000 \\
\hline Poeciludae & & & & & & & \\
\hline mosquitofish & & 0.00 & & & & & 000 \\
\hline Athennidae & & & & & & & \\
\hline brook sılversıde & 000 & 0.00 & & & & & 000 \\
\hline Percichthyıdae & & & & & & & \\
\hline stnped $x$ white bass & 013 & & 017 & & & & 0.08 \\
\hline white bass & 166 & 315 & 278 & 014 & 0.01 & & 189 \\
\hline Centrarchıdae & & & & & & & \\
\hline black crappie & 1.35 & 254 & 109 & 026 & & 017 & 122 \\
\hline bluegill & 181 & 204 & 286 & 022 & 088 & 1.07 & 1.88 \\
\hline bluegıll $\times$ green sunfish & & 002 & 014 & & & 0.03 & 0.05 \\
\hline green sunfish & 000 & 003 & 0.85 & 015 & 014 & 134 & 0.39 \\
\hline largemouth bass & 625 & 478 & 299 & & 256 & 519 & 397 \\
\hline orangespotted sunfish & 0.00 & & 0.01 & & 000 & 0.12 & 0.01 \\
\hline pumpkinseed & 004 & & 000 & & & 005 & 0.01 \\
\hline redear sunfish & & & & & 0.01 & & 0.00 \\
\hline rock bass & & & & & & 1.67 & 014 \\
\hline smallmouth bass & & & 004 & 004 & 0.04 & 1.64 & 016 \\
\hline warmouth & 0.00 & 0.04 & & & & & 001 \\
\hline white crappie & 0.21 & 0.51 & 046 & 024 & 008 & & 032 \\
\hline Percidae & & & & & & & \\
\hline logperch & & & & 001 & & & 0.00 \\
\hline sauger & & 001 & 001 & & & & 0.01 \\
\hline walleye & & & 001 & & & & 000 \\
\hline Sciaenidae & & & & & & & \\
\hline freshwater drum & 086 & 088 & 170 & & 011 & & 089 \\
\hline Total pounds per hour & 5105 & 8301 & 3949 & 1934 & 1879 & 3465 & 47.61 \\
\hline
\end{tabular}


Table 19. Species ranked by relative abundance in pounds of fish collected per hour for 1995 . Species were added to the list in descending order of abundance until $95 \%$ of the total catch for that reach was obtained. Percentages are in parentheses.

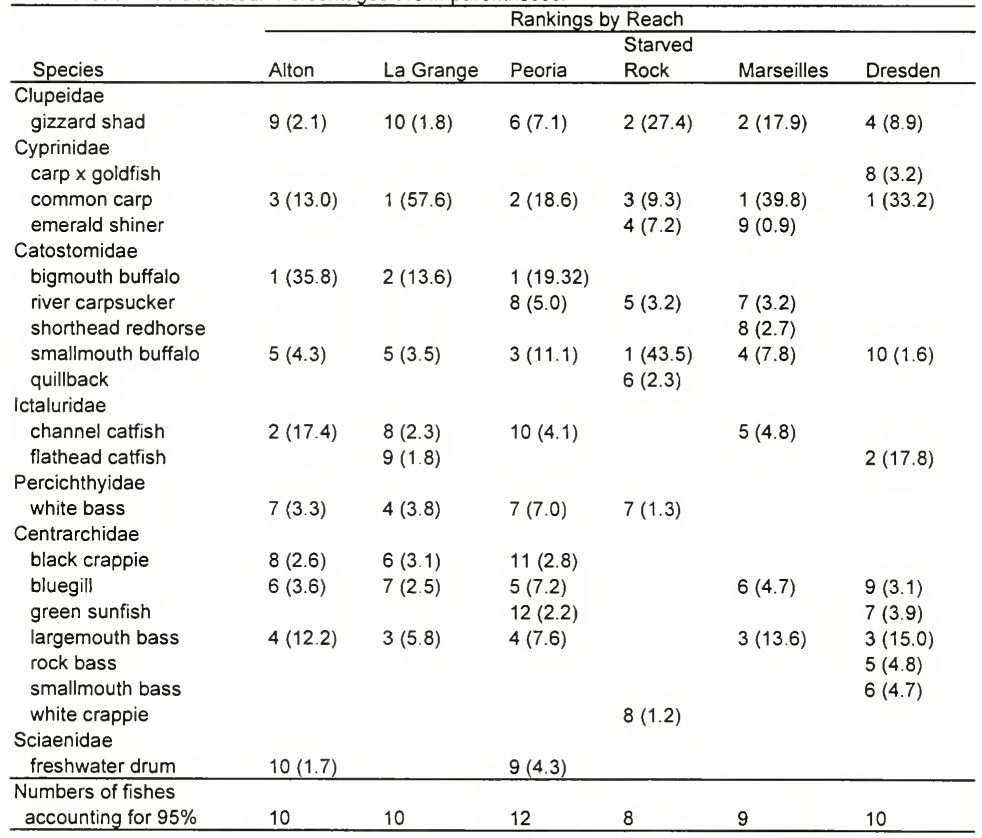


Table 20. Pounds of each fish species collected per hour of electrofishing (CPUEW) at six reaches of the IIlinois River Waterway in 1996. Pounds per hour less than 0.01 are indicated by 0.00 .

\begin{tabular}{|c|c|c|c|c|c|c|c|}
\hline \multirow[b]{2}{*}{ Species } & \multicolumn{6}{|c|}{ Reach and Hours Fished } & \multirow[b]{2}{*}{$\begin{array}{l}\text { Overall } \\
\text { CPUE } \\
2475\end{array}$} \\
\hline & $\begin{array}{r}\text { Alton } \\
500 \\
\end{array}$ & $\begin{array}{r}\text { La Grange } \\
5.50 \\
\end{array}$ & $\begin{array}{r}\text { Peoria } \\
7.00 \\
\end{array}$ & $\begin{array}{r}\text { Starved } \\
\text { Rock } \\
2.00 \\
\end{array}$ & $\begin{array}{r}\text { Marseilles } \\
225 \\
\end{array}$ & $\begin{array}{r}\text { Dresden } \\
200 \\
\end{array}$ & \\
\hline \multicolumn{8}{|l|}{ Lepisosteidae } \\
\hline shortnose gar & & 0.20 & & & & & 004 \\
\hline \multicolumn{8}{|l|}{ Clupeidae } \\
\hline gizzard shad & 0.54 & 2.48 & 3.77 & 2.88 & 1.54 & 2.73 & 2.32 \\
\hline skipjack herring & 0.09 & 0.17 & 0.05 & & & & 0.07 \\
\hline threadfin shad & 0.02 & 0.04 & 0.03 & & & 0.01 & 0.02 \\
\hline \multicolumn{8}{|l|}{ Cyprinidae } \\
\hline bluntnose minnow & & & & & & 0.01 & 0.00 \\
\hline bullhead minnow & & 0.00 & & & & & 0.00 \\
\hline carp $\times$ goldfish & & & & & 0.70 & 1.79 & 0.21 \\
\hline common carp & 15.71 & 71.15 & 1405 & 4.93 & 8.65 & 14.74 & 25.34 \\
\hline emerald shiner & 0.01 & 0.03 & 0.04 & 0.04 & 0.00 & 0.05 & 0.03 \\
\hline fathead minnow & & & 0.00 & & & & 0.00 \\
\hline golden shiner & & 0.01 & 0.02 & & & 0.01 & 0.01 \\
\hline goldfish & & 0.02 & 0.09 & & & 0.34 & 0.06 \\
\hline grass carp & 1.30 & & & & & & 026 \\
\hline red shiner & & 0.02 & 0.00 & & 0.01 & & 0.01 \\
\hline silverband shiner & & & 0.00 & & & & 0.00 \\
\hline spottail shiner & & & 0.01 & & 0.01 & 0.02 & 0.00 \\
\hline \multicolumn{8}{|l|}{ Catostomidae } \\
\hline bigmouth buffalo & 6.61 & 15.86 & 11.29 & & 2.62 & 0.48 & 8.33 \\
\hline golden redhorse & & & 0.05 & & & & 0.01 \\
\hline river carpsucker & 0.09 & 0.10 & 1.25 & 0.72 & 0.08 & & 0.46 \\
\hline shorthead redhorse & 0.65 & 0.56 & 0.25 & & 0.38 & 0.17 & 0.37 \\
\hline smallmouth buffalo & 3.38 & 2.21 & 5.81 & 8.97 & 4.19 & 0.07 & 3.93 \\
\hline \multicolumn{8}{|l|}{ Ictaluridae } \\
\hline black bullhead & & 0.00 & 0.08 & & & & 0.02 \\
\hline channel catfish & 19.06 & 5.32 & 1.57 & 0.54 & 1.25 & 1.00 & 5.71 \\
\hline flathead catfish & 0.65 & 0.14 & 0.06 & & & & 0.18 \\
\hline yellow bullhead & & & 0.00 & & & 0.14 & 0.01 \\
\hline \multicolumn{8}{|l|}{ Cyprinodontidae } \\
\hline blackstripe topminnow & & 0.00 & & & & & 0.00 \\
\hline \multicolumn{8}{|l|}{ Atherinidae } \\
\hline brook silverside & & 0.00 & & & & & 0.00 \\
\hline \multicolumn{8}{|l|}{ Percichthyidae } \\
\hline white bass & 2.09 & 2.94 & 3.88 & & & & 2.17 \\
\hline \multicolumn{8}{|l|}{ Centrarchidae } \\
\hline black crappie & 1.10 & 1.39 & 0.72 & & & & 0.73 \\
\hline bluegill & 0.79 & 0.58 & 1.17 & 0.05 & 0.44 & 0.96 & 0.74 \\
\hline bluegill $x$ green sunfish & & & 0.02 & & & & 0.01 \\
\hline green sunfish & 0.01 & 0.01 & 0.48 & & & 0.30 & 0.16 \\
\hline largemouth bass & 5.75 & 1.92 & 3.75 & 1.01 & 2.97 & 2.11 & 3.17 \\
\hline orangespotted sunfish & 0.00 & & 0.09 & & 0.07 & 0.10 & 0.04 \\
\hline rock bass & & & & & 0.16 & 0.51 & 0.06 \\
\hline smallmouth bass & & & 0.03 & 0.53 & & & 0.05 \\
\hline warmouth & & 0.09 & 0.02 & & & & 0.03 \\
\hline white crappie & 0.03 & 0.01 & 0.16 & & 0.25 & & 0.08 \\
\hline Percidae & & & & & & & \\
\hline logperch & & & 0.00 & & & & 0.00 \\
\hline mud darter & & & 0.00 & & & & 0.00 \\
\hline sauger & 0.01 & 0.14 & 0.03 & & & & 0.04 \\
\hline walleye & & & 0.01 & & & & 0.00 \\
\hline Sciaenidae & & & & & & & \\
\hline freshwater drum & 0.83 & 262 & 1.67 & & 1.26 & 0.85 & 1.40 \\
\hline Total pounds per hour & 58.74 & 108.00 & 5046 & 19.67 & 24.57 & 26.39 & 56.09 \\
\hline
\end{tabular}


Table 21. Species ranked by relative abundance in pounds of fish collected per hour for 1996 .

Species were added to the list in descending order of abundance until $95 \%$ of the total catch for that reach was obtained. Percentages are in parentheses.

\begin{tabular}{|c|c|c|c|c|c|c|}
\hline \multirow[b]{2}{*}{ Species } & \multicolumn{6}{|c|}{ Rankings by Reach } \\
\hline & Alton & La Grange & Peoria & $\begin{array}{l}\text { Starved } \\
\text { Rock }\end{array}$ & Marseilles & Dresden \\
\hline \multicolumn{7}{|l|}{ Clupeidae } \\
\hline gizzard shad & & $6(2.3)$ & $5(7.5)$ & $3(14.6)$ & $5(6.3)$ & $2(10.4)$ \\
\hline \multicolumn{7}{|l|}{ Cyprinidae } \\
\hline carp $\times$ goldfish & & & & & $7(2.8)$ & $4(6.8)$ \\
\hline common carp & $2(26.7)$ & $1(65.9)$ & $1(27.9)$ & $2(25.1)$ & $1(35.2)$ & $1(55.9)$ \\
\hline grass carp & $7(2.2)$ & & & & & \\
\hline \multicolumn{7}{|l|}{ Catostomidae } \\
\hline bigmouth buffalo & $3(11.3)$ & $2(14.7)$ & $2(22.4)$ & & $4(10.7)$ & $9(1.8)$ \\
\hline river carpsucker & & & $9(2.5)$ & $5(3.7)$ & & \\
\hline $\begin{array}{l}\text { smallmouth buffalo } \\
\text { ictaluridae }\end{array}$ & $5(5.8)$ & & $3(11.5)$ & $1(45.6)$ & $2(17.0)$ & \\
\hline channel catfish & $1(32.5)$ & $3(4.9)$ & $8(3.1)$ & & $6(5.1)$ & $5(3.8)$ \\
\hline \multicolumn{7}{|l|}{ Percichthyidae } \\
\hline white bass & $6(3.6)$ & $5(2.7)$ & $4(7.7)$ & & & \\
\hline \multicolumn{7}{|l|}{ Centrarchidae } \\
\hline black crappie & $8(1.9)$ & & & & & \\
\hline bluegill & & $4(3.7)$ & $10(2.3)$ & & & $6(3.7)$ \\
\hline largemouth bass & $4(9.8)$ & & $6(7.4)$ & $4(5.1)$ & $3(12.1)$ & $3(8.0)$ \\
\hline rock bass & & & & & & $8(1.9)$ \\
\hline \multicolumn{7}{|l|}{ Sciaenidae } \\
\hline freshwater drum & $9(1.4)$ & & $7(3.3)$ & & $6(5.1)$ & $7(3.2)$ \\
\hline \multicolumn{7}{|l|}{ Number of fishes } \\
\hline accounting for $95 \%$ & 9 & 6 & 10 & 5 & 8 & 9 \\
\hline
\end{tabular}


Table 22 Pounds of each fish species collected per hour of electrofishing (CPUE $W$ ) at six reaches of the Illinois RIver Waterway in 1997. Pounds per hour less than 0.01 are indicated by 0.00 .

\begin{tabular}{|c|c|c|c|c|c|c|c|}
\hline 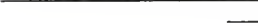 & & & ch and $\mathrm{H}$ & rs Fished & & & \\
\hline & & & & Starved & & & Overall \\
\hline & Alton & La Grange & Peoria & Rock & Marseilles & Dresden & CPUE \\
\hline Species & 5.00 & 550 & 7.25 & 2.00 & 2.25 & 2.00 & 25.00 \\
\hline Amiidae & & & & & & & 0.00 \\
\hline bowfin & 0.77 & & & & & & 0.15 \\
\hline Clupeidae & & & & & & & 0.00 \\
\hline gizzard shad & 0.56 & 1.21 & 2.03 & 1.32 & 0.90 & 1.06 & 1.24 \\
\hline skipjack herring & 0.19 & 0.10 & 0.05 & & 005 & & 0.08 \\
\hline threadfin shad & 000 & & & & 0.03 & & 000 \\
\hline Cyprinidae & & & & & & & 0.00 \\
\hline bluntnose minnow & & & & 0.05 & 002 & 0.33 & 0.03 \\
\hline bullhead minnow & & & 0.01 & 0.01 & 0.01 & & 0.00 \\
\hline central stoneroller & & & & 0.01 & 0.00 & 0.02 & 0.00 \\
\hline common carp & 29.69 & 41.83 & 27.13 & & 9.94 & 13.82 & 25.01 \\
\hline common carp $\times$ goldfish & & & 0.20 & & & & 0.06 \\
\hline emerald shiner & 0.07 & 0.03 & 0.09 & 0.17 & 0.20 & 0.04 & 0.08 \\
\hline golden shiner & & 0.00 & 0.00 & & & 0.03 & 0.00 \\
\hline goldfish & & & 0.01 & & & 047 & 0.04 \\
\hline grass carp & & & 3.19 & & & & 0.93 \\
\hline red shiner & 0.01 & & 0.00 & 0.04 & 0.12 & & 0.02 \\
\hline silver chub & & 0.00 & 0.00 & & & & 0.00 \\
\hline spottail shiner & & & 0.01 & 0.01 & 0.00 & & 0.00 \\
\hline Catostomidae & & & & & & & 0.00 \\
\hline bigmouth buffalo & 30.89 & 18.20 & 40.49 & & & & 21.93 \\
\hline golden redhorse & & 0.09 & 0.04 & & 0.18 & 0.25 & 0.07 \\
\hline river carpsucker & 0.00 & 0.06 & 1.34 & & 0.49 & & 0.45 \\
\hline shorthead redhorse & 0.01 & 0.16 & 0.88 & 0.05 & & 0.07 & 0.30 \\
\hline smallmouth buffalo & 4.52 & 3.75 & 13.29 & 7.69 & 209 & 296 & 6.62 \\
\hline Ictaluridae & & & & & & . & 0.00 \\
\hline channel catfish & 12.39 & 5.41 & 2.94 & 0.91 & 3.10 & 1.73 & 5.01 \\
\hline flathead catfish & 3.08 & 0.14 & 0.30 & & & 1.90 & 0.89 \\
\hline freckled madtom & & 0.00 & & & & & 0.00 \\
\hline tadpole madtom & & & & & 0.00 & & 0.00 \\
\hline Percichthyidae & & & & & & & 0.00 \\
\hline striped bass $x$ white bass & & 0.61 & & & & & 0.13 \\
\hline white bass & 2.91 & 3.99 & 8.16 & & & & 3.83 \\
\hline Centrarchidae & & & & & & & 0.00 \\
\hline black crappie & 1.59 & 1.37 & 1.94 & 0.17 & 0.07 & 0.96 & 1.28 \\
\hline bluegill & 027 & 1.40 & 1.85 & 0.02 & 050 & 1.61 & 1.07 \\
\hline bluegill $\times$ green sunfish & & & 0.01 & 0.01 & 0.01 & 0.29 & 0.03 \\
\hline green sunfish & 0.01 & 0.02 & 0.28 & 0.01 & 0.13 & 0.70 & 0.16 \\
\hline green $x$ orangespotted sunfish & & & 0.02 & & & & 0.00 \\
\hline largemouth bass & 2.64 & 1.22 & 6.10 & 0.63 & 1.00 & 2.94 & 2.94 \\
\hline longear sunfish & & & & & & 0.05 & 0.00 \\
\hline orangespotted sunfish & 0.00 & 0.01 & 0.09 & & & 001 & 003 \\
\hline pumpkinseed & & & 0.00 & & & & 0.00 \\
\hline rock bass & & & & & & 0.40 & 0.03 \\
\hline smallmouth bass & 0.08 & & 0.07 & & 046 & 0.25 & 0.10 \\
\hline white crappie & 0.57 & 0.46 & 0.23 & & 0.31 & & 0.31 \\
\hline Percidae & & & & & & & 0.00 \\
\hline logperch & & & 0.00 & & & & 0.00 \\
\hline sauger & & 0.04 & 001 & & & & 0.01 \\
\hline Sciaenidae & & & & & & & 0.00 \\
\hline freshwater drum & 1.38 & 1.12 & 3.40 & & 0.76 & & 1.58 \\
\hline Total pounds per hour & 91.65 & 81.24 & 114.17 & 11.06 & 20.37 & 29.84 & 74.42 \\
\hline
\end{tabular}


Table 23. Species ranked by relative abundance in pounds of fish collected per hour for 1997 . Species were added to the list in descending order of abundance until $95 \%$ of the total catch for that reach was obtained. Percentages are in parentheses.

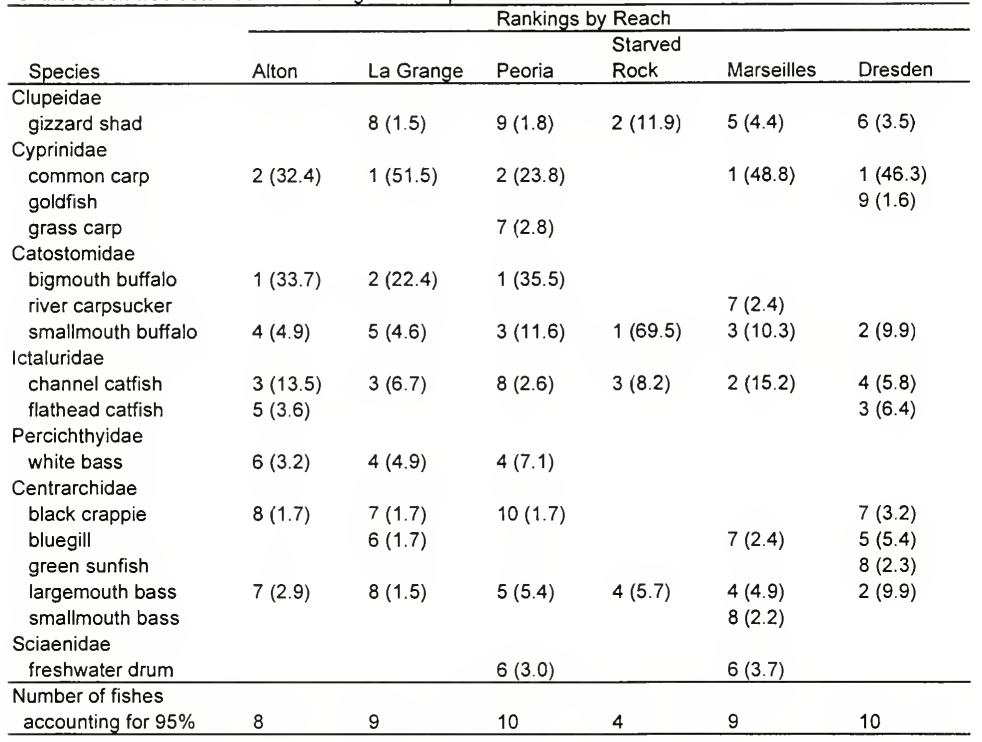


Table 24 Pounds of each fish species collected per hour of electrofishıng (CPUE $E_{W}$ ) at six reaches of the llinois River Waterway in 1998 Pounds per hour less than 001 are indicated by 000

\begin{tabular}{|c|c|c|c|c|c|c|c|}
\hline \multirow[b]{4}{*}{ Species } & \multicolumn{6}{|c|}{ Reach and Hours Fished } & \multirow{4}{*}{$\begin{array}{l}\text { Overall } \\
\text { CPUE } \\
2600\end{array}$} \\
\hline & \multirow[b]{2}{*}{ Alton } & \multicolumn{4}{|c|}{ Starved } & \multirow{3}{*}{$\begin{array}{r}\text { Dresden } \\
175 \\
\end{array}$} & \\
\hline & & La Grange & Peoria & Rock & Marseilles & & \\
\hline & 500 & 550 & 800 & 200 & 275 & & \\
\hline \multicolumn{8}{|l|}{ Clupeidae } \\
\hline gizzard shad & 031 & 127 & 251 & 1.68 & 220 & 216 & 161 \\
\hline skıpjack herning & & 001 & 001 & 0.05 & & & 001 \\
\hline threadfin shad & 002 & 001 & 002 & & & & 001 \\
\hline \multicolumn{8}{|l|}{ Hiodontidae } \\
\hline goldeye & & 013 & & & & & 0.03 \\
\hline \multicolumn{8}{|l|}{ Cyprinidae } \\
\hline bullhead minnow & 000 & & 000 & 001 & 000 & 000 & 0.00 \\
\hline bluntnose minnow & & 000 & 000 & 001 & 002 & 002 & 0.00 \\
\hline common carp & 21.95 & 5025 & 1828 & 229 & 1310 & 1111 & 22.78 \\
\hline common carp $\times$ goldfish & & & 0.33 & & & & 0.10 \\
\hline emerald shiner & 002 & 000 & 001 & 0.35 & 017 & 002 & 005 \\
\hline golden shiner & & & & & & 0.05 & 0.00 \\
\hline goldfish & & 005 & 006 & & & & 003 \\
\hline grass carp & 029 & & 180 & & & & 0.61 \\
\hline red shıner & 0.00 & 0.00 & & & & & 000 \\
\hline sand shiner & & & 000 & & & & 0.00 \\
\hline silver chub & & & 000 & & & & 000 \\
\hline silverband shıner & 0.00 & 0.00 & & & & & 0.00 \\
\hline spotfin shiner & 000 & & & 002 & 005 & & 001 \\
\hline spottail shiner & & & 001 & 003 & 0.00 & & 001 \\
\hline \multicolumn{8}{|l|}{ Catostomidae } \\
\hline bigmouth buffalo & 10.24 & 12.75 & 17.97 & & & & 1019 \\
\hline black buffalo & & 013 & 0.35 & & & & 014 \\
\hline golden redhorse & & & 020 & 0.36 & 0.71 & 048 & 020 \\
\hline quillback & & 028 & & 232 & & 029 & 026 \\
\hline river carpsucker & 041 & 047 & 213 & & 040 & & 0.88 \\
\hline shorthead redhorse & & 034 & 040 & & & & 0.20 \\
\hline smallmouth buffalo & 093 & 4.69 & 1115 & 1990 & 676 & 757 & 7.36 \\
\hline white sucker & & 0.01 & & & & & 000 \\
\hline \multicolumn{8}{|l|}{ Ictalundae } \\
\hline channel catfish & 887 & 526 & 2.80 & 210 & 029 & 494 & 421 \\
\hline flathead catfish & 147 & 2.80 & 480 & & & & 2.35 \\
\hline \multicolumn{8}{|l|}{ Cyprinodontidae } \\
\hline blackstripe topmınnow & 0.00 & & & & & 001 & 0.00 \\
\hline \multicolumn{8}{|l|}{ Poeclliidae } \\
\hline mosquitofish & & & 000 & & & & 000 \\
\hline \multicolumn{8}{|l|}{ Percichthyıdae } \\
\hline white bass & 242 & 3.70 & 411 & 064 & 022 & & 258 \\
\hline yellow bass & & 001 & & & & & 000 \\
\hline Centrarchidae & & & & & & & \\
\hline black crappie & 0.01 & 042 & 214 & 069 & 028 & & 083 \\
\hline bluegill & 0.14 & 051 & 149 & 0.08 & 010 & 059 & 065 \\
\hline bluegill $\times$ green sunfish & 0.00 & & 014 & & & 0.46 & 008 \\
\hline bluegill $x$ orangespotted sunfish & & & & & 0.01 & 001 & 000 \\
\hline green sunfish & 000 & 0.00 & 055 & 0.06 & 011 & 140 & 028 \\
\hline largemouth bass & 046 & 1.49 & 4.94 & 162 & 1.33 & 039 & 222 \\
\hline orangespotted sunfish & 0.00 & & 004 & & & 0.02 & 0.01 \\
\hline pumprinseed & & & & & 002 & & 000 \\
\hline smallmouth bass & & & 0.00 & 0.01 & & 003 & 0.00 \\
\hline warmouth & & 003 & 001 & & & & 001 \\
\hline white crappie & & 0.03 & 0.35 & & & & 0.11 \\
\hline Percidae & & & & & & & \\
\hline mud darter & 000 & & & & & & 0.00 \\
\hline sauger & 0.02 & 0.15 & 0.06 & & & & 005 \\
\hline slenderhead darter & & & 000 & & & & 000 \\
\hline Sciaenidae & & & & & & & \\
\hline freshwater drum & 0.75 & 3.71 & 284 & 0.15 & 011 & 247 & 199 \\
\hline Total pounds per hour & 4831 & 8852 & 7948 & 3232 & 2588 & 3203 & 5985 \\
\hline
\end{tabular}


Table 25. Species ranked by relative abundance in pounds of fish collected per hour for 1998 . Species were added to the list in descending order of abundance until $95 \%$ of the total catch for that reach was obtained. Percentages are in parentheses.

\begin{tabular}{|c|c|c|c|c|c|c|}
\hline \multirow[b]{2}{*}{ Species } & \multicolumn{6}{|c|}{ Rankings by Reach } \\
\hline & Alton & La Grange & Peoria & \multicolumn{2}{|l|}{ Starved } & Dresden \\
\hline \multicolumn{7}{|l|}{ Clupeidae } \\
\hline $\begin{array}{l}\text { gizzard shad } \\
\text { Cyprinidae }\end{array}$ & & & $9(3.2)$ & $5(5.2)$ & $3(8.5)$ & $5(6.7)$ \\
\hline common carp & $1(45.4)$ & $1(56.8)$ & $1(23.0)$ & $3(7.1)$ & $1(506)$ & $1(347)$ \\
\hline grass carp & & & $12(2.3)$ & & (1) & $1(04.7)$ \\
\hline \multicolumn{7}{|l|}{ Catostomidae } \\
\hline bigmouth buffalo & $2(21.2)$ & $2(14.4)$ & $2(22.6)$ & & & \\
\hline golden redhorse & & & & & $5(2.7)$ & $8(1.5)$ \\
\hline quillback & & & & $2(7.2)$ & & \\
\hline river carpsucker & & & $11(2.7)$ & & $6(1.5)$ & \\
\hline smallmouth buffalo & $6(1.9)$ & $4(5.3)$ & $3(14.0)$ & $1(61.6)$ & $2(26.1)$ & $2(23.6)$ \\
\hline \multicolumn{7}{|l|}{ Ictaluridae } \\
\hline channel catfish & $3(18.4)$ & $3(5.9)$ & $8(3.5)$ & $4(6.5)$ & $7(1.1)$ & $3(15.4)$ \\
\hline flathead catfish & $5(3.0)$ & $7(3.2)$ & $5(6.0)$ & & & \\
\hline \multicolumn{7}{|l|}{ Percichthyidae } \\
\hline white bass & $4(5.0)$ & $6(4.2)$ & $6(5.2)$ & & & \\
\hline Centrarchidae & & & & & & \\
\hline black crappie & & & $10(2.7)$ & $7(2.1)$ & & \\
\hline bluegill & & & & & & $7(1.9)$ \\
\hline green sunfish & & & & & & $6(4.4)$ \\
\hline largemouth bass & & $8(1.7)$ & $4(6.2)$ & $6(5.0)$ & $4(5.1)$ & \\
\hline \multicolumn{7}{|l|}{ Sciaenidae } \\
\hline freshwater drum & & $5(4.2)$ & $7(3.6)$ & & & $4(7.7)$ \\
\hline $\begin{array}{l}\text { Number of fishes } \\
\text { accounting for } 95 \%\end{array}$ & 6 & 8 & 12 & 7 & 7 & $11 . .7$ \\
\hline
\end{tabular}


19-26) reaches of the upper river.

In the following data summary, discussion is restricted to species that each separately accounted for over $10 \%$ of the total catch and to species that were of special significance. A $95 \%$ list was produced for each reach, in which species were ranked by relative abundance (pounds per hour) and added to the list until $95 \%$ of the total catch rate for that reach was obtained. Overall, these data indicate that in terms of weight the fish communities of the Illinois River continue to be dominated by common carp, bigmouth buffalo, and channel catfish in the lower and middle river, and common carp, smallmouth buffalo, gizzard shad, channel catfish, and largemouth bass in the upper waterway. Common carp ranked first by relative abundance in pounds of fish collected per hour in Alton, La Grange, Marseilles, and Dresden reaches in 1994; La Grange, Marseilles, and Dresden reaches in 1995; La Grange, Peoria, Marseilles, and Dresden reaches in 1996; La Grange, Marseilles, and Dresden reaches in 1997; and Alton, La Grange, Peoria, Marseilles, and Dresden reaches in 1998 (Tables 17, 19, 21, 23, and 15). Bigmouth buffalo ranked first by relative abundance in pounds of fish collected per hour in Peoria Reach in 1994 and in Alton and Peoria reaches in 1995 and in 1997 (Tables 17, 19, and 23). Channel catfish ranked first by relative abundance in pounds of fish collected per hour in Alton Reach in 1996 (Table 21). Smallmouth buffalo ranked first by relative abundance in pounds of fish collected per hour in Starved Rock Reach during all five years of this project (1994-1998). 


\section{E. Details of 1998 Sampling}

In 1998 we collected a total of 4,001 fish representing 44 species (plus three hybrids) from eleven families during $26.00 \mathrm{~h}$ of sampling at 26 sites on the Illinois Waterway and a single site on the Mississippi River (Appendices B-E). Gizzard shad was the most abundantly collected species, representing $29.0 \%$ of the total catch, followed by emerald shiner (11.2\%), bluegill (9.6\%), freshwater drum $(7.9 \%)$, common carp $(6.9 \%)$, and white bass $(6.2 \%)$. Gizzard shad were collected at all 27 sites and common carp and bluegill were collected at 26 sites. The sample from Lambie's Boat Harbor (RM170.3, Peoria Reach) yielded the most fish $(389,9.7 \%$ of the total collected from all 27 sites). The most species collected at a single site was 23 from Chillicothe (RM 180.6) in Peoria Reach. The fewest species collected at a single site was seven from Johnson Island (RM 249.6) in Marseilles Reach.

\section{CONCLUSIONS}

Our electrofishing collections on the Illinois River Waterway during August and September 1994-1998 documented the continuing recovery of the system's biological integrity. Once dominated by introduced and relatively pollution tolerant species such as common carp and goldfish (Lerczak and Sparks 1994), the Illinois River now supports a diverse assemblage of fishes, many of which support economically important sport fisheries. Ninety-four species and six hybrids have been collected since William Starrett began this survey in 1957. Seventy species 
and four hybrids have been documented by project F-101-R sampling (1989present); 44 species and three hybrids from eleven families were collected during $26.00 \mathrm{~h}$ of sampling in 1998. One species, the freckled madtom (a single specimen), was collected for the first time during project F-101-R sampling along the waterway; it was taken at Sugar Creek Island on La Grange Reach (middle river) in 1997. Also not collected previously was a green sunfish $\mathrm{x}$ orangespotted sunfish hybrid, found at the Lower Twin Sister site (RM 202.8) in 1997. We continue to document the relatively low abundance of common carp in Starved Rock Reach; this species has ranked extremely low in terms of catch rate in numbers in Marseilles and Dresden Reaches for several years. Goldfish, which were abundant in our samples in 1989 (82 individuals were collected) occurred only infrequently at sites in 1997 and 1998 (5 individuals were collected each year). Small minnow species, such as bluntnose minnow, bullhead minnow, emerald shiner, and red shiner, were extremely low in abundance in the upper waterway in 1996. However, sampling in 1997 and 1998 indicates these species are once again numerous in these reaches.

We noticed a high degree of variability in species richness among sites and also among river reaches. Some of this variability can be explained by sampling duration (site comparisons) or the number of sites sampled (reach comparisons), but there is also evidence some of our sites are inherently lower in species richness than others. For example, at most sites we have collected an average of $14-16$ species during the ten years of project F-101-R sampling. However, at Hennipin (RM 207.6), 
Pekin (RM 155.1), and Turkey Island (RM 148.0) the average has been 11 species (Appendix F). It also should be noted from Appendix $F$ that low numbers of species typically occurred at sites following the drought years of the late 1980s (1989 and 1990), while high species richness at sites typically occurred following a high water year (1995). In 1998, the greatest number of species (39) was collected from Peoria Reach and the fewest species (14) were collected from Starved Rock Reach (Appendices $\mathrm{D}$ and $\mathrm{E}$ ). The high richness of Peoria Reach is likely due, in part, to its position along the waterway which includes the Great Bend (above Hennepin) of the Illinois River. This reach represents a transition from a river which is constricted, lacks contiguous backwaters, and is high in gradient (upper river) to a large river floodplain system with low gradient (lower river) (Sparks 1977); species typical of both the upper and lower waterway have been collected and are common in Peoria Reach.

The total weight of fishes collected 1994-1998 was also highest in Peoria Reach, where CPUE ${ }_{W}$ was 114.14 (Table 8). Species accounting for this high catch in weight were bigmouth buffalo, common carp, smallmouth buffalo, and white bass. Catch in weight was also high in La Grange and Alton Reaches. For example, of 1,860 pounds of fish collected during our 1997 survey, 1,732 pounds $(93 \%)$ were collected from the lower and middle river, and only 128 pounds $(7 \%)$ were collected from the upper river. These catches reflect the high productivity of the lower and middle Illinois River floodplain ecosystem. 
Sportfishes were collected throughout the waterway in during all five years of this project (1994-1998), although catch rate in number and weight varied among reaches. For channel catfish, we collected more individuals and pounds per hour in the Alton Reach (lower river) than in the middle or upper river reaches (Tables 6-15). The white bass, however, were most abundant and provided the highest CPUE $E_{W}$ in the middle river; $\mathrm{CPUE}_{\mathrm{N}}$ was typically highest in La Grange Reach. Centrarchids such as black crappie were most abundant in the middle river reaches. Bluegill CPUE $_{N}$ was greatest in the upper waterway, although CPUE ${ }_{W}$ was typically highest in Peoria Reach of the middle river. Largemouth bass CPUE $_{N}$ has been greatest in Peoria Reach of the middle river. As in previous years of project F-101-R sampling, we collected only low numbers of smallmouth bass and sauger from the Illinois River Waterway, probably due to the locations of our sites, mostly in relatively shallow side channels behind islands. 


\section{LITERATURE CITED}

Koel, T.M., R.E. Sparks, and K.D. Blodgett. 1998. The long-term Illinois River fish population monitoring program. Project F-108-R-9 Annual Report. Center for Aquatic Ecology Technical Report 98/8. Illinois Natural History Survey, Champaign. $35 \mathrm{pp}$.

Koel, T.M., R.E. Sparks, K.D. Blodgett, and S.D. Whitney. 1997. The long-term Illinois River fish population monitoring program (F-101-R-8). Annual Report to the Illinois Department of Natural Resources. Aquatic Ecology Technical Report 97/14. Illinois Natural History Survey, Champaign. 35 pp.

Kofoid, C.A. 1903. Plankton studies. IV. The plankton of the Illinois River, 18941899 , with introductory notes upon the hydrography of the Illinois River and its basin. Part I. Quantitative investigations and general results. Illinois State Laboratory of Natural History Bulletin 6(2):95-635.

Lerczak, T.V., R.E. Sparks, and K.D. Blodgett. 1993. The long-term Illinois River fish population monitoring program (F-101-R). Annual Report to the Illinois Department of Conservation. Aquatic Ecology Technical Report 93/3. Illinois Natural History Survey, Champaign. $76 \mathrm{pp}$.

Lerczak, T.V. and R.E. Sparks. 1994. Fish populations in the Illinois River. Pages 239-241 in K.P. Pabich, editor. The changing Illinois environment: critical trends, volume 3, ecological resources. ILENR/RE-EA-95/05. Illinois Department of Energy and Natural Resources, Springfield.

Lerczak, T.V., R.E. Sparks, and K.D. Blodgett. 1994. The long-term Illinois River fish population monitoring program (F-101-R). Final Report to the Illinois Department of Conservation. Aquatic Ecology Technical Report 94/5. Illinois Natural History Survey, Champaign. $105 \mathrm{pp}$.

Lerczak, T.V., R.E. Sparks, and K.D. Blodgett. 1995. The long-term Illinois River fish population monitoring program (F-101-R-6). Annual Report to the Illinois Department of Conservation. Aquatic Ecology Technical Report 95/4. Illinois Natural History Survey, Champaign. $50 \mathrm{pp}$.

Lerczak, T.V., R.E. Sparks, and K.D. Blodgett. 1996. The long-term Illinois River fish population monitoring program (F-101-R-7). Annual Report to the Illinois Department of Natural Resources. Aquatic Ecology Technical Report 96/2. Illinois Natural History Survey, Champaign. $38 \mathrm{pp}$. 
Pflieger, W.L. 1975. The fishes of Missouri. Missouri Department of Conservation. $343 \mathrm{pp}$.

Robins, C.R., R.M. Bailey, C.E. Bond, J.R. Brooker, E.A. Lachner, R.N. Lea, and W.B. Scott. 1991. Common and scientific names of fishes from the United States and Canada. Special Publication number 20. American Fisheries Society, Bethesda, MD.

Sparks, R.E. 1977. Environmental inventory and assessment of navigation pools 24, 25, and 26, Upper Mississippi and lower Illinois Rivers: an electrofishing survey of the Illinois River, Special Report No. 5 Water Resources Center, University of Illinois, Urbana. $82 \mathrm{pp}$.

Sparks, R.E. and W.C. Starrett. 1975. An electrofishing survey of the Illinois River, 1959-1974. Illinois Natural History Survey Bulletin 31:317-380.

Sparks, R.E. and T.V. Lerczak. 1993. Recent trends in the Illinois River indicated by fish populations. Aquatic Ecology Technical Report 93/16. Illinois Natural History Survey, Champaign. $34 \mathrm{pp}$. 
APPENDIX A. Fish species collected during Long-term Resource Monitoring of the Ill inois Waterway, 1957-1998. Common names marked by an asterisk indicate species that were collected from 1989 through 1998 during federal aid project F-101-R. Common and scientific names are from Robins et al. (1991). Habitat associations are based on behavioral descriptions from Pflieger (1975) and communications with INHS fisheries biologists.

$\begin{array}{ll}\text { Lepisosteidae } & \text { longnose gar* } \\ \text { shortnose gar* } & \text { spotted gar* }\end{array}$

Ami idae

bowf in*

Hi odont idae

goldeye* mooneye*

Anguill ịdae

American eel

clupeidae

Cyprinidae

Catostomidae gizzard shad* skipjack herring* threadf in shad*

bigmouth shiner* bluntnose minnow* bul thead minnow* common carp* common carp $x$ goldf i sh* central stoneroller* common shiner creek chub emerald shiner* fathead minnow* ghost shiner golden shiner* goldf ish* grass carp* hornyhead chub Mississippi silvery minnow pugnose minnow red shiner* redfin shiner ribbon shiner river shiner* sand shiner* spotfin shiner silver chub* silverband shiner* silverjaw minnow spottail shiner* steel color shiner striped shiner suckermouth minnow*

bigmouth buffalo*

black buffalo* black redhorse golden redhorse* highfin carpsucker* northern hog sucker* quill lback*

river carpsucker* river redhorse* shor thead redhorse* silver redhorse smallmouth buffalo* white sucker
Lepisosteus osseus

Lepisosteus platostomus

Lepisosteus oculatus

Amia calva

Hiodon alosoides

Hiodon tergisus

Anquilla rostrata

Dorosoma cepedianum

Alosa chrysochloris

Dorosoma petenense

Notropis dorsalis

Pimephales notatus

Pimephales vigilax

Cyprinus carpio

Cyprinus carpio $x$ Carassius auratus

Campostoma anomalum

Luxilus cornutus

Semotilus atromaculatus

Notropis atherinoides

pimephales promelas

Notropis buchanani

Notemigonus crysoleucas

Carassius auratus

Ctenopharyngodon idella

Nocomis biguttatus

Hybognathus nuchal is

Opsopoeodus emiliae

Cyprinella lutrensis

Lythrurus umbratilis

Lythrurus fumeus

Notropis blennius

Notropis stramineus

Cyprinella spiloptera

Macrhybopsis storeriana B

Notropis shumardi

Notropis buccatus

Notropis hudsonius

Cyprinel la whipplei

Luxilus chrysocephalus

Phenacobius mirabilis

Ictiobus cyprinel lus

lctiobus niger

Moxostoma duquesnei

Moxostoma erythrurum

Carpiodes velifer

Hypentel ium niaricans

Carpiodes cyprinus

Carpiodes carpio

Moxostoma carinatum

Moxostoma macrolepidotum

Moxostoma anisurum

lctiobus bubalus

Catostomus commersoni
B

B

8

B

8

B

B

B

B 
Appendix A. Cont inued.

Ictaluridae

black bullhead* blue catfish brown bulthead* channel catfish* $f l$ athead cat $f$ ish* freckled madtom* tadpole madtom white catfish yellow bul thead*

Esocidae

grass pickerel* northern pike

Salmonidae

rainbow trout

Percopsidae

trout-perch

Cyprinodont idae

Poeciliidae

blackstripe topminnow*

Atherinidae

western mosquit tof ish *

Percichthyidae

brook silverside*

striped bass

striped bass $x$ white bass*

white bass*

white perch*

yellow bass*

Centrarchidae

black crappie*

bluegill*

green sunf ish*

green sunfish $x$

bluegill*

green sunfish $x$ orangespotted sunfish*

green sunfish $x$ pumpk inseed

largemouth bass*

longear sunfish *

orangespotted sunf ish * orangespotted sunfish $x$ bluegill

pumpkinseed*

redear sunfish*

rock bass*

smallmouth bass*

spotted sunfish*

warmouth*

white crappie*

Percidae

bluntnose darter

johnny darter

logperch*

mud darter*

sauger*

slenderhead darter*

walleye*

yellow perch*

Sciaenidae
Ameiurus melas

Ictalurus furcatus

Ameiurus nebulosus

Ictalurus punctatus

Pylodictis olivaris

Noturus nocturnus

Noturus gyrinus

Ameiurus catus

Ameiurus natal is

Esox americanus vermiculatus

Esox lucius

Oncorhynchus mykiss

Percopsis omiscomaycus

Fundulus notatus

Gambusia affinis

Labidesthes sicculus

Morone saxatilis

Morone saxatilis $x$

M. chrysops

Morone chrysops

Morone americana

Morone mississippiensis

Pomoxis nigromaculatus

Lepomis macrochirus

Lepomis cyanel lus

Lepomis cyanellus $x$

L. macrochirus

Lepomis cyanellus $x$

L. humilis

Lepomis cyanellus $x$

L. gibbosus

Micropterus salmoides

Lepomis megalotis

Lepomis humilis

Lepomis humilis $x$

L. macrochirus

Lepomis gibbosus

Lepomis microlophus

Ambloplites rupestris

Micropterus dolomieu

Lepomis punctatus

Lepomis qulosus

Pomoxis annularis

Etheostoma chlorosomum B

Etheostoma nigrum

Percina caprodes B

Etheostoma asprigene

stizostedion canadense

Percina phoxocephala

stizostedion vitreum

Perca $f$ lavescens

Aplodinotus grunniens

B
B
B
B
B
B
B
B
B


APPENDIX B. Numbers of individuals of each fish species collected on the Mississippi River (Brickhouse Slough) and the lower Illinois River (Alton Reach, RM 0-80) in 1998.

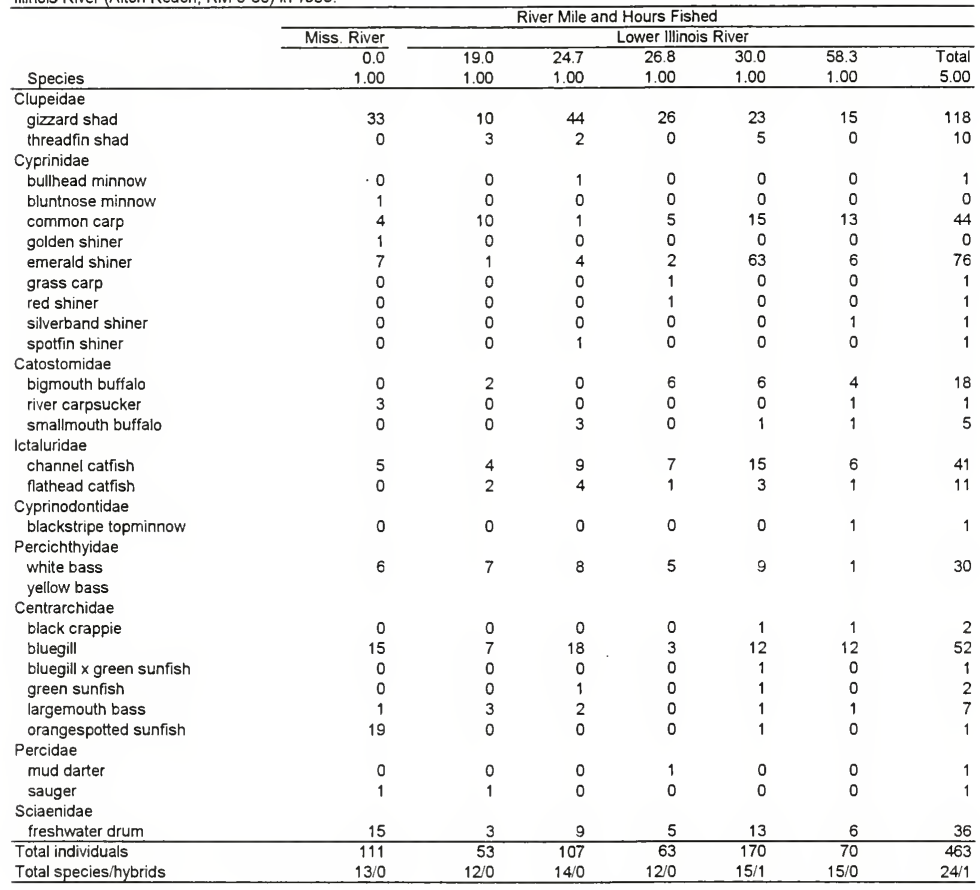


APPENDIX C Numbers of Individuals of each fish species collected on La Grange Reach (RM 80-158) of the middle IIIınols River (RM 80.231) in 1998

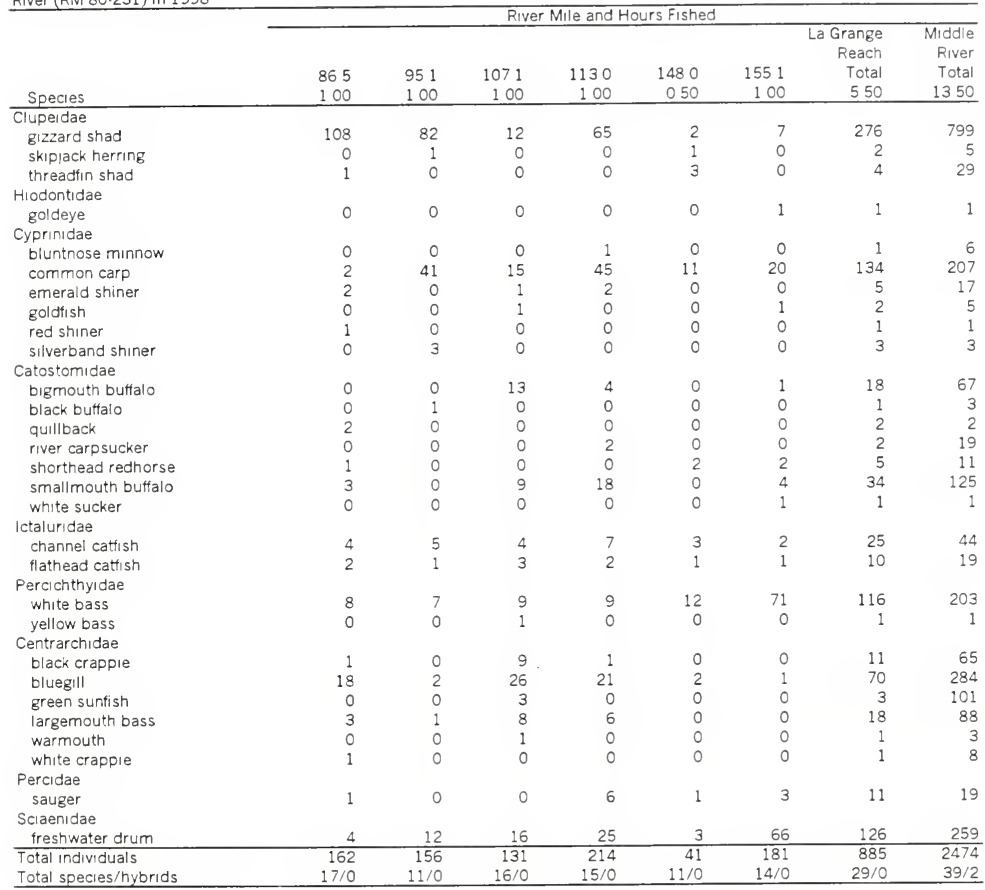


APPENDIX D Numbers of individuals of each fish species collected on Peona Reach (RM 158.231) of the middle Illinois River (RM 80.231 ) in 1998

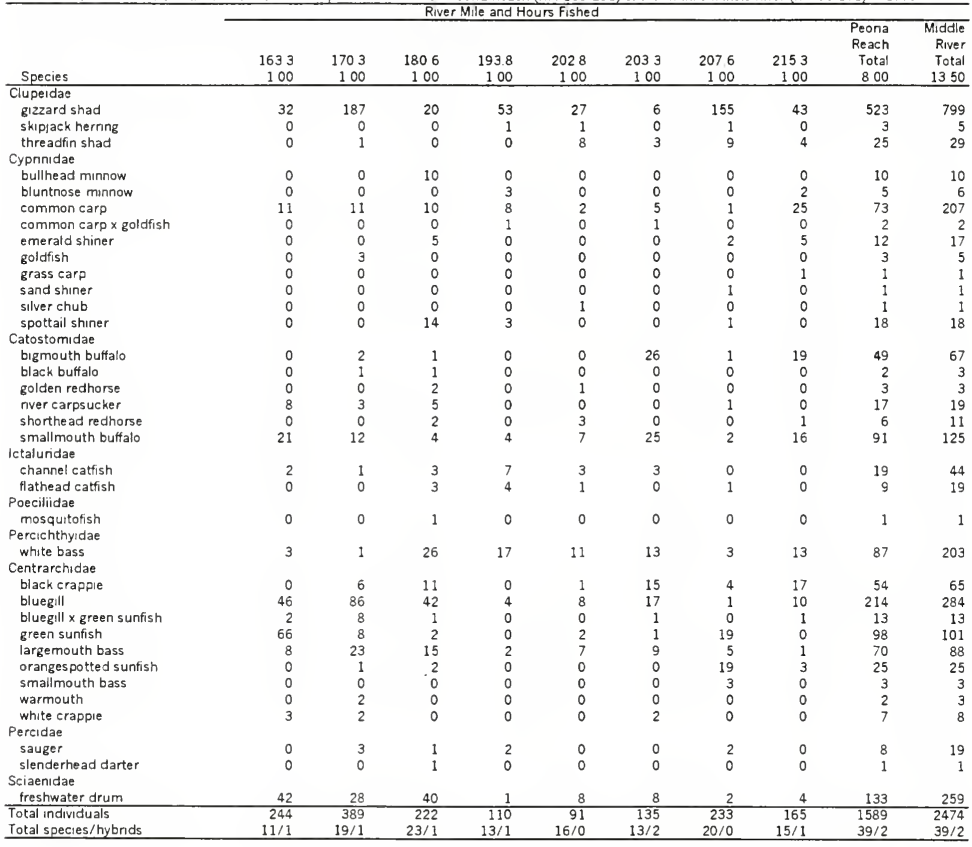


APPENDIXE Numbers of individuals of each fish species collected on Starved Rock. Marsellles, and Dresden Reaches of the upper IIlınois River waterway (RM 231.280) in 1998

\begin{tabular}{|c|c|c|c|c|c|c|c|c|}
\hline \multirow[b]{4}{*}{ Species } & \multicolumn{7}{|c|}{ River Mile and Hours Fished } & \multirow[b]{2}{*}{$\begin{array}{r}\text { Upper } \\
\text { Waterway } \\
\text { Total } \\
\end{array}$} \\
\hline & \multicolumn{2}{|c|}{ Starved Rock } & \multicolumn{3}{|c|}{ Marsellles } & \multicolumn{2}{|c|}{ Dresden } & \\
\hline & 2408 & 2415 & 2480 & 2496 & 2606 & 2773 & 2798 & \\
\hline & 100 & 100 & 100 & 075 & 100 & 075 & 100 & 650 \\
\hline \multicolumn{9}{|l|}{ Cluperdae } \\
\hline gizzard shad & 29 & 87 & 12 & 25 & 19 & 25 & 12 & 209 \\
\hline skıpjack herrıng & 0 & 1 & 0 & 0 & 0 & 0 & 0 & 1 \\
\hline \multicolumn{9}{|l|}{ Cyprinidae } \\
\hline bullhead minnow & 8 & 7 & 0 & 5 & 0 & 0 & 1 & 21 \\
\hline bluntnose minnow & 1 & 4 & 13 & 0 & 1 & 3 & 22 & 44 \\
\hline common carp & 3 & 0 & 4 & 4 & 3 & 2 & 5 & 21 \\
\hline emerald shiner & 111 & 108 & 41 & 16 & 61 & 3 & 7 & 347 \\
\hline golden shıner & 0 & 0 & 0 & 0 & 0 & 1 & 0 & 1 \\
\hline spotfın shıner & 0 & 14 & 6 & 12 & 16 & 0 & 0 & 48 \\
\hline spottail shıner & 17 & 0 & 1 & 0 & 0 & 0 & 0 & 18 \\
\hline \multicolumn{9}{|l|}{ Catostomidae } \\
\hline golden redhorse & 2 & 0 & 1 & 0 & 2 & 0 & 2 & 7 \\
\hline quillback & 0 & 6 & 0 & 0 & 0 & 1 & 0 & 7 \\
\hline river carpsucker & 0 & 0 & 0 & 0 & 1 & 0 & 0 & 1 \\
\hline smallmouth buffalo & 27 & 7 & 5 & 2 & 8 & 7 & 1 & 57 \\
\hline \multicolumn{9}{|l|}{ Ictaluridae } \\
\hline channel cattish & 1 & 1 & 0 & 0 & 1 & 2 & 3 & 8 \\
\hline \multicolumn{9}{|l|}{ Cyprinodontidae } \\
\hline blackstrıpe topminnow & 0 & 0 & 0 & 0 & 0 & 3 & 2 & 5 \\
\hline \multicolumn{9}{|l|}{ Percichthyidae } \\
\hline white bass & 0 & 4 & 2 & 2 & 0 & 0 & 0 & 8 \\
\hline \multicolumn{9}{|l|}{ Centrarchidae } \\
\hline black crappie & 2 & 1 & 3 & 0 & 0 & 0 & 0 & 6 \\
\hline bluegill & 1 & 3 & 1 & 0 & 2 & 13 & 12 & 32 \\
\hline bluegill $\times$ green sunfısh & 0 & 0 & 0 & 0 & 0 & 8 & 2 & 10 \\
\hline bluegill $x$ orangespotted sunfısh & 0 & 0 & 2 & 0 & 1 & 1 & 0 & 4 \\
\hline green sunfish & 3 & 2 & 6 & 0 & 1 & 25 & 30 & 67 \\
\hline largemouth bass & 4 & 1 & 8 & 0 & 1 & 3 & 2 & 19 \\
\hline orangespotted sunfish & 0 & 0 & 0 & 0 & 0 & 1 & 1 & 2 \\
\hline pumpkinseed & 0 & 0 & 1 & 0 & 0 & 0 & 0 & 1 \\
\hline smallmouth bass & 1 & 0 & 0 & 0 & 0 & 0 & 3 & 4 \\
\hline \multicolumn{9}{|l|}{ Sciaenıdae } \\
\hline freshwater drum & 0 & 1 & 1 & 0 & 1 & 2 & 0 & 5 \\
\hline Total individuals & 210 & 247 & 107 & 66 & 118 & 100 & 105 & 953 \\
\hline Total species/hybrids & $14 / 0$ & $15 / 0$ & $15 / 1$ & $7 / 0$ & $13 / 1$ & $14 / 2$ & $14 / 1$ & $24 / 2$ \\
\hline
\end{tabular}


APPENDIX F. Species richness (S) at Long-term Illinois River Fish Population Monitoring (F-101-R) sites.

\begin{tabular}{|c|c|c|c|c|c|}
\hline Description & Site \# & Reach & Low S (year) & High S (year) & Mean $S^{1}$ \\
\hline Treats Island & 279.8 & 3 & $11(1992)$ & $19(1995)$ & 15 \\
\hline Du Page River & 277.3 & 3 & 12 (1989 \& 1992) & 18 (1994) & 15 \\
\hline Waupecan Island & 260.6 & 4 & $11(1996)$ & 19 (1989) & 14 \\
\hline Johnson Island & 249.6 & 4 & $6 \quad(1993)$ & $16(1995)$ & 12 \\
\hline Ballards Island & 248.0 & 4 & 10 (1991) & 19 (1995) & 15 \\
\hline Bulls Island Bend & 241.5 & 5 & $8 \quad(1990)$ & 18 (1993) & 14 \\
\hline Bulls Island & 240.8 & 5 & $(1990 \& 1996)$ & 16 (1989) & 12 \\
\hline Clark Island & 215.3 & 6 & $11(1990)$ & 21 (1995) & 15 \\
\hline Hennepin & 207.6 & 6 & $2(1990)$ & 20 (1998) & 11 \\
\hline Upper Twin Sister & 203.3 & 6 & (1990) & $17(1989,94,97)$ & 14 \\
\hline Lower Twin Sister & 202.8 & 6 & (1992) & $16 \quad(1995 \&$ 1998) & 12 \\
\hline Henry Island & 193.8 & 6 & $12(1991)$ & $19(1996)$ & 15 \\
\hline Chillicothe & 180.6 & 6 & $14(1989.91,92,96)$ & 22 (1997) & 16 \\
\hline Lambie's Boat Harbor & 170.3 & 6 & $9 \quad(1989)$ & 20 (1996) & 16 \\
\hline Lower Peoria Lake & 163.3 & 6 & $10(1989)$ & 16 (1996) & 14 \\
\hline Pekin & 155.1 & 7 & $6 \quad(1992)$ & 16 (1996) & 11 \\
\hline Turkey Island & 148.0 & 7 & $9 \quad(1989 \& 1997)$ & $15(1990)$ & 11 \\
\hline Upper Bath Chute & 113.0 & 7 & $12(1994)$ & $18 \quad(1989 \& 1996)$ & 15 \\
\hline Lower Bath Chute & 107.0 & 7 & $9 \quad(1992)$ & $18(1990)$ & 15 \\
\hline Sugar Creek Island & 95.1 & 7 & $10(1989)$ & 19 (1995) & 14 \\
\hline Grape-Bar Islands & 86.5 & 7 & $7 \quad(1989)$ & $23(1994)$ & 14 \\
\hline Big Blue Island & 58.3 & 8 & $9 \quad(1990)$ & 19 (1995) & 14 \\
\hline Crater-Willow Islands & 30.0 & 8 & $12(1992 \& 1994)$ & 17 (1989) & 15 \\
\hline Hurricane Island & 26.8 & 8 & $11(1990)$ & 20 (1997) & 15 \\
\hline Dark Chute & 24.7 & 8 & 11 (1994) & $17(1990)$ & 14 \\
\hline Mortland Island & 19.0 & 8 & 11 (1989) & (1991 \& 1997) & 14 \\
\hline Brickhouse Slough & 0.0 & 26 & $10(1990)$ & $17 \quad(1991 \&$ 1995) & 15 \\
\hline
\end{tabular}

${ }^{1}$ Sites 0.0-215.3 were not sampled during 1993 ( $n=9$ years) (sites $240.8-279.8 n=10$ years). 
Appendix G (Job 5). Publications, reports, and presentations which resulted from research conducted during segments $6,7,8,9$, and 10 of project $F-101-R$, the Longterm Illinois River Fish Population Monitoring Program (funded under Federal Aid in Sportfish Restoration Act, P.L. 81-681, Dingell-Johnson, Wallop-Breaux).

\section{Publications}

Koel, T.M. 1998. Channel catfish (Ictalurus punctatus) in the Upper Mississippi River System. Project Status Report 98-11. U.S. Geological Survey, Environmental Management Technical Center, Onalaska, Wisconsin.

Koel, T.M., R. Sparks, and R.E. Sparks. 1998. Channel catfish in the Upper Mississippi River System. Survey Reports No. 353. Illinois Natural History Survey, Champaign.

Lerczak, T.V., R.E. Sparks, and K.D. Blodgett. 1994. Some upstream-todownstream differences in Illinois River fish communities. Transactions of the Illinois State Academy of Science 87(Supplement):53. (Abstract)

Lerczak, T.V. 1995. Fish community changes in the Illinois River, 1962-1994. American Currents (Summer Issue).

Lerczak, T.V. 1995. The gizzard shad in nature's economy. Illinois Audubon. (Summer Issue). Reprinted in Big River 2(12):1-3.

Lerczak, T.V. and R.E. Sparks. 1995. Fish populations in the Illinois River. Pages 7-9 in G.S. Farris, editor. Our living resources 1994. National Biological Survey, Washington, D.C.

Lerczak, T.V., R.E. Sparks, and K.D. Blodgett. 1995. Long-term trends (1959-1994) in fish populations of the Illinois River. Transactions of the Illinois State Academy of Science 88(Supplement):74. (Abstract)

Lerczak, T.V., R.E. Sparks, and K.D. Blodgett. 1995. Long-term trends (1959-1994) in fish populations of the Illinois River with emphasis on upstream-to-downstream trends. Proceedings of the Mississippi River Research Consortium 27:62-63.

Lerczak, T.V. 1996. Illinois River fish communities: 1960s versus 1990s. Illinois Natural History Survey Report No. 339.

Raibley, P.T., K.D. Blodgett, and R.E. Sparks. 1995. Evidence of grass carp (Ctenopharyngodon idella) reproduction in the Illinois and upper Mississippi Rivers. Journal of Freshwater Ecology 10:65-74. 
Sparks, R.E. 1995. Value and need for ecosystem management of large rivers and their floodplains. Bioscience 45:168-182.

Sparks, R.E. 1995. Environmental effects. Pages 132-162 in S.A. Changnon, editor. The great flood of 1993. University Corporation for Atmospheric Research (UCAR) and Westview Press.

\section{Technical Papers (presenter in bold)}

Koel, T.M. and R.E. Sparks. 1999. Interannual variation in catches of young-of-year fish correlated with hydrology of the Upper Mississippi River System. $47^{\text {th }}$ Annual Meeting of the North American Benthological Society, May 23-24, Duluth, Minnesota.

Koel, T.M. 1999. Changes in fish community structure: effects of hydrological variability in the Upper Mississippi River System. Presented to the Illinois Natural History Survey, Center for Aquatic Ecology, Havana Field Station Director Search Committee and Senior Staff, March 24, 1999.

Koel, T.M. 1998. Spatial and temporal variability of channel catfish populations in the Upper Mississippi River System. Illinois Department of Natural Resources LTRMP field station biannual retreat, December 15, Dickson Mounds, Illinois.

Koel, T.M. 1998. Long Term Resource Monitoring Program Showcase: analysis of catfish catch. Environmental Management Program Coordinating Committee, Fall Quarterly Meeting, November 19-20, Rock Island, Illinois.

Koel, T.M. and K.D. Blodgett. 1998. Fish-environment associations: effects of interannual hydrological variability on fish populations of the Illinois River waterway, 1957-1997. Upper Mississippi River Conservation Committee, Fish Technical Section Annual Fall Meeting, September 15-17, Dubuque, lowa.

Koel, T.M., K.S. Irons, T.M. O'Hara, K.D. Blodgett, and R.E. Sparks. 1998. Changes in fish community structure: effects of hydrological variability in the Upper Mississippi River System. 128th Annual Meeting of the American Fisheries Society. August 23-27, Hartford, Connecticut.

Koel, T.M., T.M. Mihuc, R.E. Sparks, and K.D. Blodgett. Upper Mississippi River System status and trends report. Fish species-environment relationships: LTRMP data analysis and preliminary results. 54th Annual Meeting of the Upper Mississippi River Conservation Committee, Moline, Illinois, 17-19 March 1998.

Blodgett, K.D. and T.M. Mihuc. Decision support using Long Term Resource Monitoring Program component data and supplementary data on the Illinois River. 54th Annual Meeting of the Upper Mississippi River Conservation Committee, Moline, Illinois, 17-19 March 1998. 
Koel, T.M. and T.M. Mihuc. Fish abundance in the La Grange Reach of the Illinois River correlated with environmental factors: problems of cross-component analysis. Presented at the Long Term Resource Monitoring Program Annual Winter Meeting, Davenport, lowa, 13 January 1998.

Lerczak, T.V., R.E. Sparks, and K.D. Blodgett. Some upstream-to-downstream differences in Illinois River fish communities. Contributed paper presented at the Illinois State Academy of Science Annual Meeting, Galesburg, Illinois, 7 October 1994.

Sparks, R.E. Large river-floodplain ecosystems of the Midwest: status, trends, and management needs. Presented at the U.S. Environmental Protection Agency's "Ecological Seminar Series" held in Chicago, Illinois, 14 March.

\section{II. Poster Presentations (presenter in bold)}

Koel, T.M. and R.E. Sparks. 1998. The Long-term Illinois River Fish Population Monitoring Program. National Meeting of the Ecological Society of America, August 10-14, Spokane, Washington.

Lerczak, T.V., R.E. Sparks, and K.D. Blodgett. Long-term trends (1959-1993) in fish populations of the Illinois River. Poster presented at the 56th Midwest Fish and Wildlife Conference, Indianapolis, Indiana, 4-7 December 1994.

Lerczak, T.V., R.E. Sparks, and K.D. Blodgett. Long-term trends (1959-1994) in fish populations of the Illinois River. Poster presented at the Illinois State Academy of Science Annual Meeting, Charleston, Illinois, 6 October 1995.

Lerczak, T.V., R.E. Sparks, and K.D. Blodgett. Long-term trends (1959-1994) in fish populations of the Illinois River with emphasis on upstream-to-downstream differences. Poster presented at the annual meeting of the Mississippi River Research Consortium, La Crosse, Wisconsin, 26-28 April 1995.

\section{Popular Presentations}

Lerczak, T.V. Wintering bald eagles along the Illinois River and factors affecting their environment. Invited presentation to the Peoria Audubon Society, Peoria, Illinois, 8 March 1995.

Lerczak, T.V. Seminar on Illinois River environmental issues. Conducted for Biology 140 (Human Ecology) at Spoon River College, 27 June 1994.

Lerczak, T.V. A photo trip up the Illinois River. After dinner talk presented to Havana Rotary Club, Havana, Illinois, 17 April 1995. 
Blodgett, K.D. Ecosystem management for the Illinois River: can biological integrity be restored? Invited lecture for Earth Day celebration at Spoon River College, Canton, Illinois, 19 April 1995.

\section{Data Requests}

1. Sam Cull, City of Peru, Electric Department, Peru, Illinois

2. Stanley and Associates, Muscatine, lowa

3. U.S. Army Corps of Engineers, Rock Island

4. Shelly Miller, Aquatic Ecologist, The Nature Conservancy, Peoria

5. K. Douglas Blodgett, Project Manager, The Nature Conservancy, Havana

6. Kevin Irons, Fishery Biologist, LTRMP, Havana 

